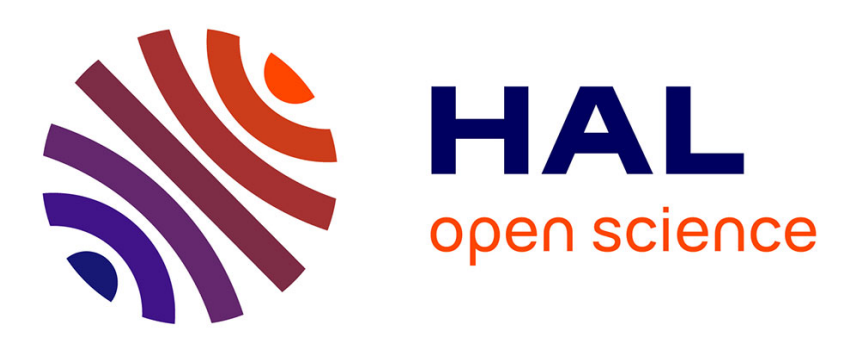

\title{
Vibroacoustics of orthotropic plates ribbed in both directions: Application to stiffened rectangular wood panels
}

\author{
Benjamin Trévisan, Kerem Ege, Bernard Laulagnet
}

\section{To cite this version:}

Benjamin Trévisan, Kerem Ege, Bernard Laulagnet. Vibroacoustics of orthotropic plates ribbed in both directions: Application to stiffened rectangular wood panels. Journal of the Acoustical Society of America, 2016, 139 (1), pp.227-246. 10.1121/1.4939706 . hal-01260373

\section{HAL Id: hal-01260373 https://hal.science/hal-01260373}

Submitted on 22 Jan 2016

HAL is a multi-disciplinary open access archive for the deposit and dissemination of scientific research documents, whether they are published or not. The documents may come from teaching and research institutions in France or abroad, or from public or private research centers.
L'archive ouverte pluridisciplinaire HAL, est destinée au dépôt et à la diffusion de documents scientifiques de niveau recherche, publiés ou non, émanant des établissements d'enseignement et de recherche français ou étrangers, des laboratoires publics ou privés. 


\title{
Vibroacoustics of orthotropic plates ribbed in both directions: application to stiffened rectangular wood panels.
}

\author{
Benjamin Trévisan ${ }^{\text {a)}}$, Kerem Ege and Bernard Laulagnet \\ Laboratoire Vibrations Acoustique \\ INSA-Lyon \\ Bâtiment St. Exupéry \\ 25 bis av. Jean Capelle, \\ F-69621 Villeurbanne cedex - France
}

a) e-mail: benjamin.trevisan@insa-lyon.fr 


\begin{abstract}
This paper is focused on the vibroacoustic behavior of a rectangular ribbed wood panel. This is done by developing an analytical model based on a variational approach, taking into account the kinetic and strain energies of a special orthotropic plate, 11 ribs oriented in a first direction and one other strong stiffener oriented in the perpendicular direction, which are considered as beams tied to the plate. A modal decomposition is adopted on the basis of the simply supported orthotropic plate. This allows calculating the modes of the wood panel (ribbed modes) in the frequency range $[0 ; 5000] \mathrm{Hz}$. The acoustical radiation of the baffled panel is also calculated. The radiation coefficients of the ribbed modes are presented and compared, when possible, to similar unribbed plate modes. Finally, the vibroacoustic analysis of the structure shows that an excitation placed on the hard point makes the panel particularly radiative and decreases the apparent critical frequency.
\end{abstract}


B. Trévisan, Kerem Ege and Bernard Laulagnet, JASA, p. 3

\section{Introduction}

With their reduced weight and their high rigidity, stiffened panels are increasingly used in many industrial domains as aircraft manufacturing, naval (submarine shells) and automotive industries (rear and side walls of trucks cabs) but also in musical instrument making (piano or guitar soundboards for example). Contrary to more simple structures like beams or plates for which the vibro-acoustic behavior is now well described ${ }^{1 ; 2 ; 3 ; 4 ; 5 ; 6 ; 7 ; 8 ; 9 ; 10}$, the design and modeling of ribbed structure with optimized vibroacoustic performances remains a major industrial challenge and a hot topic of research.

Since last decades several studies in various domains have concerned the vibroacoustic behavior of ribbed structures; most of them have been interested in periodically isotropic ribbed flat plates ${ }^{11 ; 12 ; 13 ; 14 ; 15 ; 16 ; 17 ; 18 ; 19 ; 21}$, curved panels ${ }^{22}$, stiffened cylindrical shells $^{23 ; 24 ; 25 ; 26 ; 27 ; 28 ; 29 ; 30}$. Nowadays, the development of composite materials offers new perspectives of improvements and a resurgence of interest for these structures. Moreover, the actual numerical resources make possible to describe many kinds of geometry and to include new material aspects (orthotropy by example) in order to have the more accurate

results (see ${ }^{31}$ for example). However, the effects of the broadband vibroacoustic behavior of these structures is not well known yet. In addition of the geometrical aspects, the current studies include the special characteristics of materials like interactions between the 
B. Trévisan, Kerem Ege and Bernard Laulagnet, JASA, p. 4

different layers in the case of laminated composite panels ${ }^{32 ; 33}$ or the influence of air cavities on wave propagation in the case of sandwich structures ${ }^{34 ; 35 ; 36 ; 37}$. On the particular case of ribbed wood panels, the pseudo-periodic parallel stiffening creates many localized phenomena in some specific frequency range (well described for the piano soundboard case $^{38 ; 39 ; 40}$ for example).

In order to understand the vibro-acoustic phenomena of this kind of ribbed structure, we develop a quasi-analytical model based on a variational approach and the judicious use of the associated orthotropic plate modes. We consider in this paper a simple geometry with: a thin flat plate made of wood (spruce) with a special orthotropy, pseudo-periodically ribbed in the direction of weak Young's modulus by eleven ribs and by one straight rib in the perpendicular direction (strong Young's modulus direction). The geometry is described in Fig. 1. This approach is an alternative to a complete and more accurate numerical modeling method, i.e the Finite Element Method for the structure and the Boundary Element Method for the acoustical radiation. In order to understand the vibroacoustic phenomena of stiffened structures, we assume that a method that avoids any discretization of the object would contribute to knowledge on the subject and would allow for easy parametric studies (influence of the ribs, their number, their width/length). 
B. Trévisan, Kerem Ege and Bernard Laulagnet, JASA, p. 5

The first part of the article presents the quasi-analytical model and discusses modal aspects due to stiffening on the whole frequency range $[0,5000] \mathrm{Hz}$. Then in a second part, the modal radiation coefficients of a ribbed structure that have never been shown before are given. Finally, in the last part of the article the numerical results show that the apparent critical frequency of a heterogeneous structure is very sensitive to the excitation point of the effort. In particular, when applied on a hard point of the structure, i.e. on the straight rib, it decreases the apparent critical frequency, selecting the more radiative modes.

\section{Vibratory modeling}

\section{A. Context of the study}

In this study, we focus on a rectangular ribbed wood panel (see Fig. 1). We consider a special orthotropic plate (axis of orthoropy parallel to the edges, see Fig. 1). The boundary conditions are simply supported and the plate has one straight rib in the direction of the strong Young's modulus $\vec{x}$ and eleven pseudo-periodic smaller ribs in the perpendicular direction $\vec{y}$. These superstructures are also made of spruce.

The eccentricity of the different superstructures is taken into account. Moreover, the thickness of the plate, width and height of the superstructures are constant along their length.

The analytical model developed is based on a variational approach that draws its 
B. Trévisan, Kerem Ege and Bernard Laulagnet, JASA, p. 6

inspiration from Laulagnet \& Guyader's works ${ }^{23 ; 41}$. Moreover, the traversal displacement is developed on the basis of simply supported plate modes.

It is necessary to calculate the kinetic and strain energies of the whole structure to calculate the Hamilton function of the problem, and develop it on the basis mentioned above in order to write a matrix problem and, finally, determine a new specific basis for the ribbed structure.

\section{B. Hamilton function for eleven stiffeners and one straight rib}

The modeling is based on a variational approach. So, we want to express the Hamilton functional of the whole problem, which is defined as:

$$
H=\int_{t_{0}}^{t_{1}}\left(E_{c}-E_{d}+E_{w}\right) d t
$$

with $\left[t_{0} ; t_{1}\right]$ an arbitrary time interval, $E_{c}$ and $E_{d}$ the kinetic and strain energies and $E_{w}$, the work done by the external force or sound pressure. In order to solve the eigenvalue problem, $E_{w}$ is equal to zero for the moment.

\section{Kinetic hypothesis and boundary conditions at the interface plate / superstructures}

Because the thickness of the plate is very small compared to the other dimensions, the Love-Kirchhoff hypothesis referring to thin plates was adopted. Thus pumping effects and shearing in the two bending planes are ignored ${ }^{5}$. The middle plane of the plate does not 
accept motion in the direction of the plate edges.

Taking into account these hypotheses and writing the motion as linear into the thickness (limited development at order 1), leads to the following motion field:

$$
\left\{\begin{array}{l}
u(x, y, z, t)=-z \frac{\partial w}{\partial x} \\
v(x, y, z, t)=-z \frac{\partial w}{\partial y} \\
w(x, y, z, t)=w(x, y, t)
\end{array}\right.
$$

with $z \in\left[-\frac{h}{2} ; \frac{h}{2}\right]$ ( $h$ being the plate thickness) and where $u(x, y, z, t)$ and $v(x, y, z, t)$ are the motions in directions $\vec{x}$ and $\vec{y}$ and $w(x, y, t)$ is the plate transversal displacement.

The different superstructures are considered as beams driven by bending and torsion coupled to the plate. Nevertheless, superstructures cannot be approached as simple beams due to their coupling with the plate. Indeed, boundary conditions are applied to the plate, thus its motion governs all the elements attached to it, as shown in Fig. 2 which illustrates this phenomenon in bending and torsion motions.

Therefore displacements and rotations are considered as continuous at each plate / stiffener interface. Moreover, the negligible shearing effects of the Love-Kirchhof plate theory are propagated to the ribs through the continuities mentioned above. Likewise for the other kinetic hypothesis, like the pumping effect and shell assumptions applied to the 
displacement field of the superstructure. This hypothesis is in agreement with the idea of considering the ribs and straight rib as beams.

After performing calculus, the following displacement field is obtained for ribs oriented in direction $\vec{y}$ at position $x=x_{r}$ :

$$
\left\{\begin{array}{l}
u_{r}(y, z, t)=-z w_{, x}\left(x_{r}, y, t\right) \\
v_{r}(y, z, t)=-z w_{, y}\left(x_{r}, y, t\right) \\
w_{r}(x, y, t)=w\left(x_{r}, y, t\right)+\left(x-x_{r}\right) w_{, x}\left(x_{r}, y, t\right)
\end{array}\right.
$$

where $x \in\left[x_{r}-\frac{b}{2} ; x_{r}+\frac{b}{2}\right]$ and $z \in\left[\frac{h}{2} ; \frac{h}{2}+H\right]$ and where $h$ and $H$ are respectively the plate thickness and rib height, and $b$ its width.

\section{Expression of the Hamilton function as a function of the kinetic field of the plate}

We express the Hamilton functions of each sub-part on the basis of the different fields of motion. These functions are expressed by the integral of the difference between the kinetic and strain energies on an arbitrary time interval (see Appendix I for more information on this calculus).

Thus we write successively the actions of the plate, of the bending and torsion of a rib in direction $\vec{y}$ at position $x=x_{r}$ and of the bending and torsion of the straight rib in direction 
$\vec{x}$ at position $y=y_{c}$ by:

$$
\begin{gathered}
H_{p}=\int_{t_{0}}^{t_{1}} \frac{1}{2} \int_{x=0}^{l} \int_{y=0}^{L} \rho h \dot{w}^{2}-\left(D_{1} w_{, x x}^{2}+D_{3} w_{, y y}^{2}+D_{2} w_{, x x} w_{, y y}+D_{4} w_{, x y}^{2}\right) d x d y d t \\
H_{r b}=\int_{t_{0}}^{t_{1}} \frac{1}{2} \int_{x=0}^{l} \int_{y=0}^{L}\left[\rho_{r}\left(I_{f} \dot{w}_{, y}^{2}+b H \dot{w}^{2}\right)-E_{r} I_{f} w_{, y y}^{2}\right] \delta\left(x-x_{r}\right) d x d y d t \\
H_{r t}=\int_{t_{0}}^{t_{1}} \frac{1}{2} \int_{x=0}^{l} \int_{y=0}^{L}\left[\rho_{r} I_{g} w_{, x}^{2}-G_{r} I_{g} w_{, x y}^{2}\right] \delta\left(x-x_{r}\right) d x d y d t \\
H_{b b}=\int_{t_{0}}^{t_{1}} \frac{1}{2} \int_{x=0}^{l} \int_{y=0}^{L}\left[\rho_{c}\left(I_{f c} \dot{w}_{, x}^{2}+b_{c} H_{c} \dot{w}^{2}\right) \cdot-E_{c} I_{f c} w_{, x x}^{2}\right] \delta\left(y-y_{c}\right) d x d y d t \\
H_{b t}=\int_{t_{0}}^{t_{1}} \frac{1}{2} \int_{x=0}^{l} \int_{y=0}^{L}\left[\rho_{c} I_{g c} w_{, y}^{2}-G_{c} I_{g c} w_{, x y}^{2}\right] \delta\left(y-y_{c}\right) d x d y d t
\end{gathered}
$$

where $\rho$ and $\rho_{r}$ are respectively the mass density of the plate and the rib $h, l$ and $L$ the thickness, the length and the width of the plate; $D_{1}=\frac{E_{x} h^{3}}{12\left(1-v_{x y} v_{y x}\right)}, D_{3}=\frac{E_{y} h^{3}}{12\left(1-v_{x y} v_{y x}\right)}$, $D_{2}=\frac{v_{y x} E_{x} h^{3}}{6\left(1-v_{x y} v_{y x}\right)}$ and $D_{4}=\frac{G_{x y} h^{3}}{3}$ the plate dynamic stiffness ; $v_{x y}$ and $v_{y x}=v_{x y} \frac{E_{y}}{E_{x}}$ Poisson's coefficients of the plate; $E_{x}$ and $E_{y}$ the two Young's modulus of the orthotropic plate ; $E_{r}$ the Young's modulus of a rib ; $b$ and $H$ respectively the width and the height of a rib ; $I_{f}$ and $I_{g}$ the rib momentum of bending and torsion inertia. Concerning the variables $(.)_{c}$, they are referring to the straight rib oriented in the direction $\vec{x}$.

The Table 1 recaps the different constants used in the previous equations.

Note that all these functions are defined on the intervals $x \in[0 ; l]$ and $y \in[0 ; L]$. This formulation does not mean that the superstructures occupy the whole area of the plate. Indeed, they are considered as punctual in their widths, justifying the Dirac distributions 
$\delta\left(x-x_{r}\right)$ and $\delta\left(y-y_{c}\right)$.

Because the rib height is low compared to its width, the torsional inertia does not take warping into account. Moreover, because the plate controls the rib motion, the action of the whole system is expressed as a function of the plates transversal displacement and its spatial and temporal derivatives.

Finally, for a structure with a determined number $N_{r}$ of ribs in direction $\vec{y}$ and a straight rib in direction $\vec{x}$, we express the entire function by:

$$
H_{\text {System }}=H_{p}+\sum_{i=1}^{N_{r}}\left(H_{r b}^{(i)}+H_{r t}^{(i)}\right)+\left(H_{b b}+H_{b t}\right)
$$

\section{Hamilton function as a function of modal amplitudes of unribbed plate modes}

We develop these energies on the basis of simply supported unribbed plate modes.

This basis, currently used in the area of vibrations ${ }^{23 ; 41}$ is particularly adapted for an analytical approach. Moreover, the radiation of the plates was studied specifically for these boundary conditions.

\section{Modal decomposition on the basis of simply supported plate modes}

Through modal decomposition, the plate transversal displacement is written as a 
linear combination of unribbed plate modes weighted by modal amplitudes $a_{m n}(t)$ :

$$
w(x, y, t)=\sum_{m=1}^{M} \sum_{n=1}^{N} a_{m n}(t) \phi_{m n}(x, y) \forall x \in[0 ; l] \& y \in[0 ; L]
$$

where $\phi_{m n}(x, y)=\sin \left(\frac{m \pi}{l} x\right) \sin \left(\frac{n \pi}{L} y\right)$.

It should be recalled that these modal shapes have orthogonal properties:

$$
\int_{0}^{l} \int_{0}^{L} \phi_{m n}(x, y) \phi_{p q}(x, y) d x d y=\left\{\begin{array}{cc}
\frac{l * L}{4} & \text { if } \mathrm{m}=\mathrm{p} \& \mathrm{n}=\mathrm{q} \\
0 & \text { if } \mathrm{m} \neq \mathrm{p} \| \mathrm{n} \neq \mathrm{q}
\end{array}\right.
$$

The modal decomposition (10) is finite; there are truncations on the orders $(\mathrm{M}, \mathrm{N})$. The further the problem is from the initial basis, meaning without superstructures, the higher the order of the truncations must be to guarantee the convergence of the numerical results. In particular, we know that the strong inter-modal coupling introduced by the superstructures will lead us to high orders $(\mathrm{M}, \mathrm{N})$.

\section{New Hamilton function as a function of the modal amplitudes of simply supported plate modes}

By injecting the modal decomposition Eq. (10) into the Hamilton function expressions Eq. (4) to (8) and using the orthogonal properties Eq. (11), it is possible to analytically calculate the surface integral in the Hamilton function. 
In this case, the functional depends on the couples of variables $\left(a_{m n}(t), \dot{a}_{m n}(t), a_{p q}(t), \dot{a}_{p q}(t)\right)$ and no longer on the transversal displacement $w(x, y, t)$ and its space and temporal derivatives $w_{, x}, w_{, x x}, w_{, y y}, w_{, x y}$ and $\dot{w}$. Thus, we have:

$$
H_{\text {System }}\left(a_{m n}(t), \dot{a}_{m n}(t), a_{p q}(t), \dot{a}_{p q}(t)\right)=\int_{t_{0}}^{t_{1}} \mathcal{L}\left(a_{m n}(t), \dot{a}_{m n}(t), a_{p q}(t), \dot{a}_{p q}(t)\right) d t
$$

where $\mathcal{L}\left(a_{m n}(t), \dot{a}_{m n}(t), a_{p q}(t), \dot{a}_{p q}(t)\right)$ is termed the Lagrangian of the system.

Afterwards, the action of the system is minimized following the principle of less action. In practice, the differential form of Euler-Lagrange is used to determine the evolution of the system, leading to the following equation:

$$
\delta H_{\text {System }}=0 \Leftrightarrow \frac{\partial \mathcal{L}}{\partial a_{p q}}-\frac{d}{d t} \frac{\partial \mathcal{L}}{\partial \dot{a}_{p q}}=0
$$

express for each mode of the unribbed plate and where $p=1 \rightarrow M$ and $q=1 \rightarrow N$.

This minimization leads us to a single equation for a particular mode $(\mathrm{p}, \mathrm{q})$ :

$$
\sum_{m=1}^{M} \sum_{n=1}^{N} \mathcal{M}(m, n, p, q) \cdot \ddot{a}_{p q}+\sum_{m=1}^{M} \sum_{n=1}^{N} \mathcal{K}(m, n, p, q) \cdot a_{p q}=0
$$

where $\mathcal{M}(m, n, p, q)$ and $\mathcal{K}(m, n, p, q)$ are respectively mass and rigidity matrix terms depending on integers $(m, n, p, q)$. The sums on the modal amplitude integers highlight the inter-modal coupling introduced by the superstructures. However, this expression does not take into account the external effort at this stage of the development. 
B. Trévisan, Kerem Ege and Bernard Laulagnet, JASA, p. 13

\section{Matrix formulation of the problem}

Expressing the problem in this way makes it easier to show the coupling phenomena due to the presence of the superstructures. Finally, we obtain a homogenized problem for which the size is conditioned by an adapted truncation guaranteeing the convergence of the solution. Thus we obtain the following matrix formulation:

$$
\begin{gathered}
\left\{\left[M_{r}^{\text {plate }}\right]+\left[M_{r s}^{\text {ribs }}+\left[M_{r s}^{\text {straight rib }}\right]\right\} \quad\left\{\ddot{a}_{p}\right\}\right. \\
+\left\{\left[K_{r}^{\text {plate }}\right]+\left[K_{r s}^{\text {ribs }}+\left[K_{r s}^{\text {straight rib }}\right]\right\} \quad\left\{a_{p}\right\}=\overline{0}\right.
\end{gathered}
$$

where $r=(m, n)$ and $s=(p, q)$ and where $M$ and $K$ represent mass and rigidity matrices.

The system (15) is constituted by the sum of diagonal matrices for the plate and of full and symmetrical matrices for the superstructures. These introduce very strong coupling of the unribbed plate modes, as we will see in the following sections. Indeed, these matrices do not have a dominant diagonal.

Consequently, the size of the matrix is also important for the convergence and precision of the results. Indeed, since the wood panel studied is an heterogeneous structure, its modes are far from the initial basis (simply supported plate). Thus it is necessary to take into account modes with small wavelengths (high order modes) in order to describe its vibrational behavior correctly. In the present case, this leads us to a truncation on the 
couple (p,q) around (40,30), i.e. 1200 simply supported plate modes. In the following, when the new basis of the ribbed structure is obtained, these 1200 modes will obtain around 350 panel modes in the frequency range [0;5000] Hz.

\section{Calculus of ribbed panel modes}

Ribbed modes are not as simple as simply supported plate modes, as we will see in the following. The first step to understand the behavior of such a structure is to determine and thus calculate the new basis specific to the panel.

\section{Eigenvalue problem}

In order to determine the new ribbed basis, we are interested in the canonical form of the eigenvalue problem:

$$
(\overline{\bar{N}}-\lambda \overline{\bar{I}}) \bar{a}=\overline{0}
$$

where $\overline{\bar{N}}$ is the dynamical rigidity matrix, also called core of the system ${ }^{42}$, with $\overline{\bar{N}}=\overline{\bar{M}}^{-1} \cdot \overline{\bar{K}}$, where $\overline{\bar{K}}$ and $\overline{\bar{M}}$ are the mass and rigidity matrices of the system and with $\lambda$ the eigenvalues : $\lambda=(2 \pi f)^{2}$.

The search for the eigenvalues and eigen vectors of the $\overline{\bar{N}}$ matrix solving Eq. (16) leads to a diagonal matrix called $\overline{\bar{D}}$ with square angular frequencies and to a matrix of eigen vectors called $\overline{\bar{P}}$ whose terms are the modal amplitudes weighted by the eigen modes of the 
B. Trévisan, Kerem Ege and Bernard Laulagnet, JASA, p. 15

unribbed plate used to re-create ribbed plate modes.

$$
\overline{\bar{D}}=\left(\begin{array}{cccc}
\left(2 \pi f_{1}\right)^{2} & & & (0) \\
& & & \\
& \left(2 \pi f_{2}\right)^{2} & & \\
& & \ddots & \\
& & & \left(2 \pi f_{i}\right)^{2}
\end{array}\right) ; \quad \overline{\bar{P}}=\left(\begin{array}{cccc}
a_{11}^{(1)} & a_{11}^{(2)} & \ldots & a_{11}^{(P * Q)} \\
a_{21}^{(1)} & a_{21}^{(2)} & & a_{21}^{(P * Q)} \\
\vdots & \vdots & \ddots & \vdots \\
a_{P Q}^{(1)} & a_{P Q}^{(2)} & & a_{P Q}^{(P * Q)}
\end{array}\right)
$$

\section{Ribbed plate modes}

Each ribbed plate mode is reconstituted by a linear combination of simply supported plate modes. Thus for each eigen frequency $f_{i}$, we calculate the corresponding modal shape by:

$$
\phi^{(i)}(x, y)=\sum_{m=1}^{M} \sum_{n=1}^{N} a_{m n}^{(i)} \phi_{m n}(x, y) \forall x \in[0 ; l] \& y \in[0 ; L]
$$

\section{Numerical results: panel modes}

In the aim of applying in the future the model presented to musical instruments, and in particular to the piano soundboard, dimensions of superstructures are inspired from ${ }^{39}$ (see the perspectives in the last section of the article).For this study, the frequency range of interest is $[0 ; 5000] \mathrm{Hz}$. The modal shapes are calculated for an order (p,q) equal to $(41,27)$ to ensure the convergence of the solution.

The presence of only one straight rib in the direction $\vec{x}$ seems to separate the plate into two parts. A vibrating area and a non-vibrating area is often found on each side of this one $\left(3^{\text {th }}, 4^{\text {th }}\right.$ and $5^{\text {th }}$ mode in Fig. 3). 
B. Trévisan, Kerem Ege and Bernard Laulagnet, JASA, p. 16

Under these conditions, only the two first modes seem to be similar to the homogeneous unribbed plate modes. The separating effect appears from the third mode $(242 \mathrm{~Hz})$, splitting the plate into 2 areas; for each side the vibration is approximately homogeneous. With this consideration, the ribbed structure appears like an equivalent homogeneous unribbed plate but only over a reduced area delimited by the straight rib. Similarly, the regular addition of stiffeners causes localized vibrations. However, due to these several additional ribs, the split areas are smaller than those with the straight rib along $\vec{x}$ and this phenomenon does not appear in the first modes. Thus Fig. 3 shows that when the frequency increases, modes can be seen for which the vibration is delimited over a small surface, for example the $17^{\text {th }}$ mode. At high frequency, the modes become very complex. A large number of localized vibratory phenomena can be seen such as oblique waves and wave reflections in the inter-rib spaces. See Fig. 3.

\section{Acoustic radiation of ribbed panel}

The behavior of such a structure is not limited to a purely vibratory aspect. Its radiation, which is complex due to its particular geometry, has rarely been studied with the Finite Element Method, due to high numerical and temporal costs (Chabassier \& al. ${ }^{31}$ in the case of a grand piano soundboard). This is why the analytical model presented here is a good alternative in order to perform parametric studies quickly and with minimum computer resources. 
In the modeling, the baffled hypothesis is made. In real situation, a panel radiates in the two half spaces (up and down) which naturally communicate, tending at low frequency to considerable acoustical short cuts. Indeed, the radiation becomes dipolar as the wavelengths are large compared to the size of the structure. Thus it is better to assume no baffle in this frequency range. The influence of the baffle on the acoustic radiation at low frequency can be found in ${ }^{3 ; 9}$.

Nevertheless, when focusing on a frequency higher than the first octave, the acoustic wavelengths fall and become smaller than the size of the panel and the baffled hypothesis becomes solid. Therefore only the radiation on one face is calculated.

We are interested in the acoustic radiation of the ribbed wood panel and more particularly in the general indicators such as the acoustical radiated power $W(\omega)$, the space average quadratic velocity $\left\langle v^{2}\right\rangle(\omega)$ and the radiation coefficient $\sigma(\omega)$. We invite the reader to refer to the Table 2 to read the rest of this paper.

\section{A. Vibroacoustic problem: forced response}

In the following, we considered a sinusoidal and harmonic excitation applied at $\left(x_{e}, y_{e}\right)$ with an amplitude $F$ of $1 \mathrm{~N}$. So, the Hamilton function now includes the work done by this external force minimized following the same approach that in the previous parts. It comes the vector of generalized effort $\bar{F}_{\text {gen }}$ and these components are defined by the following 
relation:

$$
F_{p q}=\frac{\partial E_{w}}{\partial a_{p q}}=\frac{\partial F \sum_{m} \sum_{n} a_{m n} \phi_{m n}\left(x_{e}, y_{e}\right)}{\partial a_{p q}}=F \phi_{p q}\left(x_{e}, y_{e}\right)
$$

with $a_{p q}$ an arbitrary mode of the series. Thus, in the frequency domain, the problem with a second member and the vector of modal amplitudes can be written with the following expressions:

$$
\begin{gathered}
\left(\overline{\bar{K}}^{*}-\omega^{2} \overline{\bar{M}}\right) \bar{a}=\bar{F}_{g e n} \\
\Leftrightarrow \bar{a}=\left(\overline{\bar{K}}^{*}-\omega^{2} \overline{\bar{M}}\right)^{-1} \bar{F}_{g e n}
\end{gathered}
$$

Note that the light fluid assumption is made. Therefore the Eq. (20 \& 21) does not take into account the acoustical boundary pressure that we assume to be negligible at the first order. For a full calculus, see $^{3}$.

Moreover the structural damping is taken into account by making the stiffness matrix $\overline{\bar{K}}^{*}=\overline{\bar{K}} *(1+j \eta)$ as a complex number with $\eta=2 \%$ for all the frequencies.

\section{B. Expression of the ribbed panel radiated power}

In the case of a harmonic problem, after application of the modal decomposition (10), we give the following expression of the radiated power ${ }^{6}$ :

$$
W(\omega)=\frac{1}{2} \Re\left\{-j \omega \sum_{p} \sum_{q} a_{p q}^{*}(\omega) P_{p q}^{a}(\omega)\right\}
$$

where $a_{p q}^{*}(\omega)$ is the conjugated modal amplitude of a mode $(\mathrm{p}, \mathrm{q})$ and $P_{p q}^{a}(\omega)$ the 
generalized boundary pressure defined by:

$$
P_{p q}^{a}(\omega)=\int_{S_{p}} \phi_{p q}(x, y) p(x, y, z=0) d S
$$

with $p(x, y, z=0)$, the boundary pressure.

Solving the Helmoltz equation in the wave number domain ${ }^{3 ; 43}$ and applying the boundary conditions (baffled plate), we obtain an expression of the pressure in the wave number domain:

$$
\tilde{p}\left(k_{x}, k_{y}, z\right)=\frac{j \rho_{0} \omega^{2} \tilde{u}_{z}\left(k_{x}, k_{y}, z=0\right)}{k_{z}} e^{-j \cdot k_{z} \cdot z} \text { avec } k_{x}, k_{y} \in \Re^{2}
$$

where $k_{z}=\sqrt{k^{2}-k_{x}^{2}-k_{y}^{2}}$ is the reduced wave number in the direction $\vec{z}$ and $\tilde{u}_{z}\left(k_{x}, k_{y}, z=0\right)$ the particular acoustical displacement Fourier transform of the plate.

With the series development of the plate Eq. (10), we obtain:

$$
\tilde{u}_{z}\left(k_{x}, k_{y}, z=0\right)=\sum_{m} \sum_{n} a_{m n} \tilde{\phi}_{m n}\left(k_{x}, k_{y}\right)
$$

where $\tilde{\phi}_{m n}\left(k_{x}, k_{y}\right)$ refers to the Fourier transform of a simply supported mode. Returning to the spatial domain, using an inverse Fourier transform leads to the following expression of the pressure in the fluid:

$$
p(x, y, z)=\frac{1}{4 \pi^{2}} \iint_{-\infty}^{+\infty} \frac{j \rho_{0} \omega^{2}}{k_{z}} \sum_{m} \sum_{n} a_{m n} \tilde{\phi}_{m n}\left(k_{x}, k_{y}\right) e^{+j k_{x} x} e^{+j k_{y} y} e^{-j k_{z} z} d k_{x} d k_{y}
$$


B. Trévisan, Kerem Ege and Bernard Laulagnet, JASA, p. 20

In this expression, note the undefined double integral in the wave number domain.

Therefore it is estimated numerically ${ }^{3}$. By integrating this expression of the pressure in Eq. (23), we define the generalized acoustic pressure:

$$
P_{p q}^{a}=j \omega \sum_{m} \sum_{n} a_{m n} Z_{m n p q}(\omega)
$$

where $Z_{m n p q}(\omega)$ is the inter-modal radiation impedance of modes $(\mathrm{m}, \mathrm{n})$ and $(\mathrm{p}, \mathrm{q})$ with:

$$
Z_{m n p q}(\omega)=\frac{\rho_{0} \omega}{4 \pi^{2}} \iint_{-\infty}^{+\infty} \frac{\tilde{\phi}_{m n} \tilde{\phi}_{p q}^{*}}{\sqrt{k^{2}-k_{x}^{2}-k_{y}^{2}}} d k_{x} d k_{y}
$$

where ${ }^{*}$ is the conjugate complex number.

Neglecting inter-modal coupling, i.e. $Z_{m n p q}=0$ if $n m \neq p q$, thus the classical light fluid assumption (effects of inter-modal coupling can be consulted in ${ }^{8 ; 18 ; 32 ; 44}$ ), we can define a new expression of the acoustic radiated power of the panel in the frequency domain as ${ }^{6}$ :

$$
W(\omega)=\frac{\omega^{2}}{2} \sum_{m} \sum_{n}\left|a_{m n}\right|^{2} R_{m n m n}(\omega)
$$

where $R_{m n m n}(\omega)=\Re\left(Z_{m n m n}(\omega)\right)$. The $R_{m n m n}(\omega)$ calculus is performed numerically. We show that ${ }^{3 ; 6}$ :

$$
R_{m n m n}(\omega)=\frac{\rho_{0} \omega}{4 \pi^{2}} \iint_{-k}^{k} \frac{\left|\tilde{\phi}_{m n}\right|^{2}}{\sqrt{k^{2}-k_{x}^{2}-k_{y}^{2}}} d k_{x} d k_{y}
$$

C. Expressions of the space average quadratic velocity $\left\langle v^{2}\right\rangle(\omega)$ and of the radiation coefficient $\sigma(\omega)$ 
The space average quadratic velocity expression is given by ${ }^{6}$ :

$$
<v^{2}(\omega)>=\frac{1}{2 S} \int_{x=0}^{l} \int_{y=0}^{L}\left|v(x, y, \omega)^{2}\right| d x d y
$$

where $S=l * L$ is the plate surface. Applying the modal decomposition Eq. (10) and using the orthogonal properties of the modal shapes in the simply supported basis, leads to the following expression of the space average quadratic velocity in the frequency domain:

$$
<v^{2}(\omega)>=\frac{\omega^{2}}{8} \sum_{m} \sum_{n}\left|a_{m n}(\omega)\right|^{2}
$$

Finally, we define the radiation coefficient by ${ }^{6}$ :

$$
\sigma(\omega)=\frac{1}{\rho_{0} c S} \frac{W(\omega)}{<v^{2}>(\omega)}
$$

where $\rho_{0}$ and $c$ are respectively the mass density and the celerity of sound.

\section{Expression of the radiated power in the panel basis}

Calculating the radiated power in the ribbed modes basis is not necessary but allows defining the modal radiation coefficient of a ribbed structure, which has never been presented in the literature before. We give the expression of the power Eq. (22) in scalar form:

$$
\begin{gathered}
W(\omega)=\frac{1}{2} \Re\left\{-j \omega \sum_{p} \sum_{q} a_{p q}^{*}(\omega) P_{p q}^{a}(\omega)\right\} \\
\Leftrightarrow W(\omega)=\frac{1}{2} \Re\left\{-j \omega \bar{a}^{*^{T}}(\omega) \cdot \bar{P}_{a}\right\}
\end{gathered}
$$


where $\bar{a}^{*^{T}}$ is the transposed vector of modal amplitudes taken from Eq. (21) and $\bar{P}_{a}$ is the vector of generalized boundary pressure that we defined in the same manner as:

$$
\begin{aligned}
P_{p q}^{a}= & j \omega \sum_{m} \sum_{n} a_{m n}(\omega) Z_{m n p q}(\omega) \\
& \Rightarrow \bar{P}_{a}=j \omega \overline{\bar{Z}}^{a}(\omega) \vec{a}(\omega)
\end{aligned}
$$

where $\overline{\bar{Z}^{a}}$ is the inter-modal acoustic impedance matrix whose terms are $Z_{m n p q}(\omega)$. This leads us to a compact matrix expression of the radiated power. Thus we obtain:

$$
W(\omega)=\frac{\omega^{2}}{2} \bar{a}^{*^{T}}(\omega) \cdot \overline{\bar{R}}^{a}(\omega) \cdot \bar{a}(\omega)
$$

where $\overline{\bar{R}}^{a}(\omega)$ is the real part of the simply supported plate inter-modal impedance matrix and $\bar{a}(\omega)$ the vector of modal amplitudes for an excitation at a specific frequency obtained from solving Eq. (21).

As presented in the first part, solving the homogeneous problem Eq. (15) conferred the matrix of eigenvectors $\overline{\bar{P}}$, and consequently, the transfer matrix $\overline{\bar{T}}$ to the new ribbed basis which is the orthonormed basis of eigenvectors. By definition, we have:

$$
\bar{a}=\overline{\bar{T}} \cdot \bar{b}
$$

where $\bar{a}$ is the vector of modal amplitudes in the initial plate basis and $\bar{b}$ the same vector now in the ribbed basis. Therefore we give the acoustic radiated power expression in the ribbed basis:

$$
W(\omega)=\frac{\omega^{2}}{2} \bar{b}^{* T}(\omega) \cdot \overline{\bar{T}}^{T} \cdot \overline{\bar{R}}^{a}(\omega) \cdot \overline{\bar{T}} \cdot \bar{b}(\omega)
$$


B. Trévisan, Kerem Ege and Bernard Laulagnet, JASA, p. 23

Thus we define the real part of the acoustical modal impedance in the ribbed basis by:

$$
\overline{\bar{R}}^{s}(\omega)=\overline{\bar{T}}^{T} \cdot \overline{\bar{R}}^{a}(\omega) \cdot \overline{\bar{T}}
$$

\section{E. Radiation coefficient definition of a ribbed mode}

We explained in the introduction that we neglected inter-modal coupling between simply supported plate modes, which means considering zero energy exchanges between two modes of the simply supported plate. From the matrix point of view, the inter-modal impedance matrix $\overline{\bar{R}}^{a}$ will be assumed to be diagonal.

This simplification allows easily calculating the radiation of the structure in a light fluid and introducing the radiation coefficient of a particular mode $(\mathrm{p}, \mathrm{q})$. We now want to determine if this hypothesis can be generalized to a ribbed structure, at least in a frequency range.

This raises the question of whether extra-diagonal terms (inter-modal coupling) are negligible, which implies a dominant diagonal matrix. Indeed, diagonal terms represent the interaction of a ribbed mode with itself whereas extra-diagonal terms represent energy exchanges between modes.

\section{F. Inter-modal coupling in the acoustic radiation of a ribbed structure}

In this part, we focus on the normalized radiation impedance ${ }^{8 ; 11} r(\omega)=\frac{R^{a \mid s}(\omega)}{\rho_{0} c \frac{S}{4}}$ in the frequency range $[25 ; 5000] \mathrm{Hz}$. Regarding $R^{a \| s}$, it could be $R^{a}()$ or $R^{s}()$ according to have 
B. Trévisan, Kerem Ege and Bernard Laulagnet, JASA, p. 24

interest in unstiffened coefficients or stiffened coefficients. The results will be presented as 10. $\log \left(\left|r_{i j}(\omega)\right|\right)$. When $i=j$, we obtain the real radiation impedance part of a ribbed panel mode which is what we will study in the following. When $i \neq j$, we talk about inter-modal radiation impedance between modes, an impedance which can be negative, contrary to the case $i=j$.

In the same way as for the radiation impedance of simply supported plate modes, it is common to verify if interaction terms are negligible compared to diagonal terms:

$$
r_{m n m n}(\omega)>>\left|r_{m n p q}(\omega)\right|
$$

Indeed, the radiation impedance notion of a mode $(\mathrm{m}, \mathrm{n})$ is meaningful only if these interaction terms $(\mathrm{m}, \mathrm{n}),(\mathrm{p}, \mathrm{q})$ between two modes of simply supported plate are negligible ${ }^{3 ; 6 ; 8}$.

Fig. 4-a and 4-c present the modal radiation coefficients of $1^{\text {st }}$ and $5^{\text {th }}$ ribbed panel modes (see Fig. 1) as well as examples of inter-modal coupling. Fig. 4-a shows that for the first mode, the extra-diagonal terms (dashed plots) are far below the diagonal term (full plot) with differences exceeding $10 \mathrm{~dB}$.

On the other hand, for high order modes, we quickly see that energy exchanges are strong. Even if it is negligibly higher than the coincidence frequency (here around 700/800 Hz), Fig. 4-c shows that these energy exchanges (dashed plots) have the same order of 
B. Trévisan, Kerem Ege and Bernard Laulagnet, JASA, p. 25

magnitude as the modal radiation coefficient of the mode coupled with itself (full plot) under $150 \mathrm{~Hz}$ and in the frequency range [300;500] Hz.

Nevertheless, these tendencies also exist for simply supported plate modes. Indeed, locally, the coupling terms of simply supported plate modes can be at the same order of magnitudes as coefficients with themselves, as show Fig. 4-b and 4-d.

Indeed, for the mode $(1,1)$ of a simply supported rectangular plate with the same dimensions, we also notice that differences between some couplings (dashed plots) and the radiation coefficient $r_{1,1 ; 1,1}$ (full plot) are only around $5 \mathrm{~dB}$ under $100 \mathrm{~Hz}$. See Fig. 4-b. For high order modes such as mode $(5,5)$, presented in Fig. 4-d, we note that energy exchanges are sometimes higher than the exchange of the mode $(5,5)$ with itself, from low to high frequencies. These less common results can be consulted in CVALOR ${ }^{44}$.

\section{G. Radiation coefficient of ribbed modes}

As recalled above, speaking about modal radiation coefficients supposes that modal interaction terms could be neglected initially. We make this assumption in the following,

meaning we neglect the extra-diagonal terms of the matrix $\overline{\bar{R}}^{s}(\omega)$. This allows focusing on the notion of the radiation coefficient of a ribbed mode.

\section{Plate ribbed by eleven stiffeners in one direction $\vec{x}$}


Firstly, we focus on a structure ribbed in only one direction. We take the geometry presented in Fig. 1 for which we remove the straight rib in the direction $\vec{x}$. Thus the plate is pseudo-periodically ribbed in the direction $\vec{x}$ and each rib has a different height and width with mean values around 19 and $27 \mathrm{~mm}^{39}$. Therefore the structure is quasi-homogeneous but where small variations of dimensions can lead to considerable asymmetry on the vibration ${ }^{45 ; 46}$. This is the case of the two first modes. For a simply supported plate with the same dimensions, the first mode $(1,1)$ presents a symmetry, contrary to our case study where the vibration is localized on the right half part (Fig. 5). This loss of symmetry implies a decrease of the modal radiation coefficient of the first ribbed mode compared to that of a simply supported plate mode $(1,1)$ under the coincidence frequency. But both are close as shown by the small differences, with a maximum of $1.5 \mathrm{~dB}$ at $25 \mathrm{~Hz}$.

The second ribbed plate mode is also strongly affected by small variations of rib dimensions. Although it resembles, at the first order, the mode $(2,1)$ of a simply supported plate mode, we note a considerable loss of the anti-symmetry of the latter with different magnitudes at either side. Contrary to the first radiation coefficient, which is only weakly affected, this coefficient is very sensitive at low frequency, as shown in Fig. 6. Indeed, we see differences higher than $20 \mathrm{~dB}$ between the two coefficients at $25 \mathrm{~Hz}$ and, contrary to the first mode, this time the stiffeners increase the radiation coefficient. We assume that the 
B. Trévisan, Kerem Ege and Bernard Laulagnet, JASA, p. 27

reduction of the vibrating anti-symmetry tends to make it more efficient at low frequency. These first results show the influence of small variations of rib dimensions but they also show that it is impossible to predict if the radiation increases or decreases.

At a higher frequency, we see an alternation in most cases of quasi identical non ribbed plate modes and of modes with localized vibrations. Therefore most of the radiation coefficients are similar to those of a non-ribbed plate like the $5^{\text {th }}$ mode compared in Fig. 7 to the mode $(5,1)$ of a rectangular plate with the same dimensions.

On the contrary, some modes present localized vibrations in inter-ribbed spaces. It would be pointless to compare them to the modes of an unribbed plate with the same dimensions. For the 6 th mode (Fig. 8), a comparison with the mode $(1,2)$ of a rectangular plate reduced by 4 in the direction $\vec{x}$ slightly overestimates the radiation coefficient around $4 \mathrm{~dB}$ at $25 \mathrm{~Hz}$ but shows that for some modes, only a small area of the structure radiates. Thus the radiation coefficient of a quasi homogeneous structure, pseudo-periodically ribbed in only one direction, can be close to those of an unribbed rectangular plate. In most cases, the modal shapes are similar to those of an unribbed plate and so the corresponding radiation coefficients are similar (Fig. 7). However, there are also localized modes for which the radiation coefficients are close to those of a reduced plate (Fig. 8). Finally, it is impossible to predict how the superstructures will modify the radiation because of the smallest modification of the geometry can have an considerable influence. 
B. Trévisan, Kerem Ege and Bernard Laulagnet, JASA, p. 28

\section{Ribbed plate in two perpendicular directions: complete ribbed wood panel}

Although we can assume that a structure ribbed in both directions quasi homogeneously will have a similar behavior to the previous case, this is completely different for the ribbed panel. Indeed, the straight rib introduces a strong heterogeneity which splits the panel into two vibrating areas, as seen in section II.D.3. Thus we are now interested in the radiation coefficients of the ribbed wood panel (Fig. 1).

The presence of the straight rib along $\vec{x}$ tends to regularize the small variations of different heights / widths of each rib along $\vec{y}$ and implies a symmetrization effect on the two first modes. Therefore they are similar to modes $(1,1)$ and $(2,1)$ of a rectangular unribbed plate with the same dimensions ${ }^{45 ; 4}$. Thus the conclusions are the same concerning the radiation coefficients, as shown in Fig. 9 and 10.

On the other hand, the heterogeneous aspect due to the straight rib appears from the 3th mode for which the panel is split into two. Thus the radiation coefficient is similar to that of a rectangular unribbed plate with reduced dimensions along $\vec{y}$. Indeed, the radiation coefficient of the mode $(3,1)$ of the reduced plate (dashed dark plot in Fig. 11) is a very good approximation of that of the $3^{\text {th }}$ mode (full plot). However, we note that at the first order the mode $(3,1)$ of a plate with the same dimensions (light dash-dotted plot) is also a correct approximation, as shown by the small differences, with a maximum of $2 \mathrm{~dB}$ at 25 Hz. 
B. Trévisan, Kerem Ege and Bernard Laulagnet, JASA, p. 29

Increasing the frequency considerably changes the behavior of the ribbed plate in two directions in comparison to the previous case. Indeed, reduced homogeneous modal shapes are rare from the $7^{\text {th }}$ mode which is the last distinctly identifiable mode. Fig. 12 shows a comparison between its radiation coefficient and that of mode $(2,2)$ of an unribbed plate with the same dimensions. Despite this relative agreement, this comparison shows that both coefficients are only comparable at a frequency higher than $100 \mathrm{~Hz}$. Indeed, at low frequency, the differences become very significant, reaching $20 \mathrm{~dB}$ at $25 \mathrm{~Hz}$.

For high order modes, the straight rib introduces geometric complexity, having considerable impact on the modal aspect of the system and making mode identification almost impossible. This is alternated with localized modes. The $10^{\text {th }}$ panel mode (Fig. 13) is a perfect example of this aspect. The vibration is split into two areas where we can see 4 maximum amplitudes of vibration below the straight rib and 3 above it. Consequently, its radiation coefficient is also unique and not comparable to the radiation coefficient of unribbed plate modes or that of the $271^{t h}$ mode (Fig. 14) for which the vibration is extremely complex.

Finally, the high complexity of the vibratory aspect caused by structural heterogeneity is similar to that caused to the radiation. Thus the modes quickly become dependent on the geometry of the structure, removing the pertinence of making a comparison with a simple 
B. Trévisan, Kerem Ege and Bernard Laulagnet, JASA, p. 30

structure like the rectangular simply supported plate.

\section{H. Influence of inter-modal coupling on the radiation coefficient $\sigma(\omega)$}

In view to providing an interpretation of ribbed mode radiation coefficients, we have assumed up to now that inter-modal coupling of the radiation impedance matrix was negligible. We now focus on the calculus of the radiation coefficient $\sigma(\omega)$ Eq. (33), plotted in 10. $\log (\sigma(\omega))$ for a sinusoidal excitation placed on the straight rib at the coordinates $(\mathrm{x}=$ $0.73 \mathrm{~m} ; \mathrm{y}=0.61 \mathrm{~m})$. The structure modeled refers to Fig. 1, i.e. the ribbed wood panel. Fig. 15 shows the influence of taking into account coupling terms. Globally, we observe very good agreement between the full and dashed plots, except for the frequency range $[400 ; 800] \mathrm{Hz}$. Indeed, in this range we tend to underestimate the radiation coefficient $\sigma(\omega)$ at a maximum of $2 \mathrm{~dB}$.

Globally, this result shows the bias involved when couplings are assumed to be negligible. It is localized within a short frequency range and is rather weak.

\section{Numerical comparison between several excitation points}

In this last part, we deal with the radiation of the same ribbed panel with different excitation point. Moreover, we now take into account the inter-modal coupling of the radiation impedance matrix in the ribbed basis. Since the radiation coefficient alone is 
B. Trévisan, Kerem Ege and Bernard Laulagnet, JASA, p. 31

insufficient for understanding the influence of superstructures on radiation, we consider the acoustical radiated power, the space average quadratic velocity and the radiation coefficient Eq. (29), (32) and (33) in the following.

\section{A. Influence of the excitation point: hard and weak point}

For a ribbed structure, the excitation point has a considerable influence on acoustical radiation. To show this, we chose two characteristic points on the panel. These two points (see Fig. 16) are at the abscissa $x_{e}=1 \mathrm{~m}$ and at the ordinate:

- $y_{e}=0.61 \mathrm{~m}$, on the largest superstructure (straight rib), so the hard point,

- $y_{e}=0.47 \mathrm{~m}$, on the plate far from the first point, near the middle between the edge of the plate and the straight rib.

However, first let us define the critical frequency $f_{c}$ of a plate. At this frequency, the propagation velocity of flexural waves in the plate and in the fluid (air) is the same. Below this frequency, the bending wave is subsonic and poorly radiative; higher, it is supersonic and radiative.

For a simple case like an unribbed isotropic plate, we can easily determine the propagation velocity of the flexural wave in the structure. Thus we have the following expression of the critical frequency for an isotropic plate ${ }^{2}$ :

$$
f_{c}=\frac{c^{2}}{2 \pi h} \sqrt{\frac{12 \rho\left(1-\nu^{2}\right)}{E}}
$$


B. Trévisan, Kerem Ege and Bernard Laulagnet, JASA, p. 32

depending on the velocity of propagation of the wave in the fluid $c$, the plate thickness $h$, the plate mass density $\rho$, the Young's modulus $E$ and the Poisson's coefficient $\nu$ of the plate.

For an orthotropic plate, there are two critical frequencies because of different propagation velocities due to the two Young's moduli. In our case, these frequencies are around $1450 \mathrm{~Hz}$ and $7250 \mathrm{~Hz}$. For a structure with a complex geometry and superstructures, it is impossible to calculate $f_{c}$. Nevertheless, we define the apparent critical frequency when the radiation coefficient is close to unity ( $0 \mathrm{~dB}$ in log scale).

Therefore there are two important results concerning the radiation coefficient (Fig. 17-c). Firstly, without mentioning the excitation point, we can see that the simple presence of the superstructures decreases the apparent critical frequency from $1450 \mathrm{~Hz}$ to less than $800 \mathrm{~Hz}$. Thus the superstructures increase the radiation efficiency in the frequency range [800;1450] Hz. Obviously, above $800 \mathrm{~Hz}$ all the modes excited are radiative; on the contrary, at low frequencies below $400 \mathrm{~Hz}$, the modes are weakly radiative, independently of the excitation point.

The second important result concerns the transitional frequency range [400;800] Hz which can be defined when the excitation position has an influence on the radiation efficiency. 
B. Trévisan, Kerem Ege and Bernard Laulagnet, JASA, p. 33

Thus, excitation at a hard point of the structure (straight rib) tends to increase the radiation factor in this transitional frequency range: the apparent critical frequency decreases from $800 \mathrm{~Hz}$ (dashed plot) to $400 \mathrm{~Hz}$ (full plot). Indeed, far from the straight rib, the radiation factor is low under $800 \mathrm{~Hz}$.

However, the conclusions are different for the two other indicators (Fig. 17-a and 17-b). Under $800 \mathrm{~Hz}$ the excitation point has a small influence. On the contrary, at a high frequency, we can observe that for both the acoustical radiated power and the space average quadratic velocity that the average values are around $15 \mathrm{~dB}$ lower for an excitation placed on a hard point. Obviously, the straight rib has a lower mobility which is why an excitation on the hardest point of this heterogeneous structure leads to decreasing the radiated power and the space average plate velocity.

Finally, these results show that an excitation on a hard point implies a decrease of the average velocity and of the acoustical radiated power in favor of better radiation efficiency at mid-frequency.

\section{B. Perspectives: toward the piano soundboard}

In this paper, we have mentioned the possibility to apply our model to the piano 
B. Trévisan, Kerem Ege and Bernard Laulagnet, JASA, p. 34

soundboard. The soundboard of a piano plays an essential role in the functioning of the instrument. Indeed, string sections are too thin to radiate on their own. Thus their vibrations are transmitted to the soundboard through the bridge which serves as an effective acoustic radiator. The vibroacoustic behavior of this ribbed structure has been studied by Suzuki, Conklin, Giordano, Boutillon $47 ; 48 ; 49 ; 50 ; 51 ; 53$ and more recently by Berthaut, Ege \& Boutillon and Chaigne $38 ; 39 ; 40 ; 52$.

With a work of mind, the bridge can be assimilated to the straight stiff rib of our semi-analytical model whereas the eleven smaller ribs can be assimilated to the stiffeners (See Fig. 18). Our results show that a smaller bridge could increase the acoustical power of high pitched notes but this also implies increased mobility and thus lower sustain of sound. This raises the question of the optimal dimensions of the bridge in order to find the best acoustical power/sustain trade-off.

An excitation placed on the straight rib / bridge provides another non negligible advantage for acoustical radiation of a stiffened structure: in other words the presence of this straight rib homogenizes the response of the ribbed structure. To demonstrate this, we consider an excitation moving along the straight rib and a second moving in the same direction at $y_{e}=0.47 \mathrm{~m}$ (see dashed lines on Fig. 16). Obviously, for the latter the excitation will sometimes occur on the plate, sometimes on the ribs. Fig. 19 presents the evolution of the acoustical radiated power as a function of the frequency along the $\vec{x}$ axis for these two cases. 
B. Trévisan, Kerem Ege and Bernard Laulagnet, JASA, p. 35

Fig. 19-a shows that the radiated power is globally homogenous for an excitation on the straight rib. On the contrary, it is strongly affected for an excitation far from this superstructure (Fig. 19-b). As mentioned before, for a point placed directly on the plate, the average value of the acoustical radiated power is around $15 \mathrm{~dB}$ higher than for an excitation on the straight rib / bridge. However, we can also see that the value falls when the excitation moves to the ribs. This means that the response has a large number of discontinuities. Indeed, the ribs can be seen clearly in Fig. 19-b. These results show the importance of the straight rib / bridge in order to obtain the most homogenous acoustical response possible.

\section{Conclusion}

This article has presented the basis of a new analytical model for predicting the vibroacoustic behavior of orthotropic ribbed plates. Here, we have limited our study to the case of a wood panel: a special rectangular orthotropic plate with several ribs in one direction and one perpendicular straight rib.

The model developed allowed us to present the modes of a complex structure and focuses on the acoustic radiated power, the space average quadratic velocity and the radiation coefficients for different excitation points. It also allowed determining the influence of stiffeners on acoustic radiation.

Moreover we have introduced the notion of modal radiation coefficients for a ribbed 
B. Trévisan, Kerem Ege and Bernard Laulagnet, JASA, p. 36

structure and have showed that it is meaningful. Namely the first modes of the stiffened panel exhibit radiation coefficients whose behaviour compares to the one of the unstiffened corresponding mode. On contrary, when the modal shape becomes complex, no similarity can be done with the radiation factor of existing modes and the tendency needs to be evaluated numerically, because of the specificity of the complex stiffened mode under consideration. One other conclusion is that the radiation coefficient of a stiffened panel increases sensibly, when the point excitation is located on a rib. Consequently, the apparent critical frequency decreases too in a sensible manner, rendering this critical frequency depending of the localization of the effort.

\section{Acknowledgment}

This work was performed within the framework of the Labex CeLyA of Université de Lyon, supervised by the French National Research Agency (ANR-10-LABX-0060/ ANR-11-IDEX-0007). 
B. Trévisan, Kerem Ege and Bernard Laulagnet, JASA, p. 37

\section{Appendix I : Hamilton function calculus}

In this Appendix, we give the main calculus steps for expressing the Hamilton function of the system without external force. The original expression is:

$$
H=\int_{t_{0}}^{t_{1}}\left(E_{c}-E_{D}\right) d t
$$

where $E_{c}$ refers to kinetic energy and $E_{D}$ the strain energy of the system. $\left[t_{0}, t_{1}\right]$ is an arbitrary time interval. We initially focus on the contribution of the plate and then the contributions of the superstructures.

Thus, we split the calculus into three sub-functions:

- $H_{p}$ for the plate

- $H_{r}$ for a rib in the direction $\vec{y}$

- $H_{b}$ for the straight rib in the direction $\vec{x}$

with :

$$
H_{\text {plate }}=H_{p}+\sum_{i=1}^{N_{r}} H_{r}^{(i)}+H_{b}
$$

The contribution of the plate can be found in the literature ${ }^{2}$ :

$$
H_{\text {plate }}=\int_{t_{0}}^{t_{1}} \frac{1}{2} \int_{S} \rho h \dot{w}^{2}-\left(D_{1} w_{, x x}^{2}+D_{3} w_{, y y}^{2}+D_{2} w_{, x x} w_{, y y}+D_{4} w_{, x y}^{2}\right) d S d t
$$


The result is not immediate for the superstructures. We give the displacement field of a rib in direction $\vec{y}$ at position $x=x_{r}$ :

$$
\left\{\begin{array}{l}
u_{r}(y, z, t)=-z w_{, x}\left(x_{r}, y, t\right) \\
v_{r}(y, z, t)=-z w_{, y}\left(x_{r}, y, t\right) \\
w_{r}(x, y, t)=w\left(x_{r}, y, t\right)+\left(x-x_{r}\right) w_{, x}\left(x_{r}, y, t\right)
\end{array}\right.
$$

where $x \in\left[x_{r}-\frac{b}{2} ; x_{r}+\frac{b}{2}\right]$ and $z \in\left[\frac{h}{2} ; \frac{h}{2}+H\right]$ where $h$ and $H$ are respectively the plate thickness and the rib height.

Kinetic and strain energies are calculated on the entire rib volume placed at $x=x_{r}$. Let the kinetic energy be:

$$
E_{C}=\frac{1}{2} \int_{x=-\frac{b}{2}}^{\frac{b}{2}} \int_{y=0}^{L} \int_{h=\frac{h}{2}}^{\frac{b}{2}+H} \rho_{r}\left(\dot{u}_{r f t}^{2}+\dot{v}_{r f t}^{2}+\dot{w}_{r f t}^{2}\right) d x d y d z
$$

Replacing $\dot{u}_{r f t}^{2}, \dot{v}_{r f t}^{2}$ and $\dot{w}_{r f t}^{2}$ by their expression from (45) and after making the integral on variables $\mathrm{x}$ and $\mathrm{z}$, we have:

$$
E_{C}=\frac{1}{2} \rho_{r} \int_{y=0}^{L}\left(I_{f} \dot{w}_{, y}^{2}+b H \dot{w}^{2}+I_{g} \dot{w}_{, x}^{2}\right) d y
$$

where bending inertia is equal to $I_{f}=\frac{b}{3}\left[\left(\frac{h}{2}+H\right)^{3}-\left(\frac{h}{2}\right)^{3}\right]$ and torsion inertia is $I_{g}=\frac{H b^{3}}{12}+I_{f}$ 
B. Trévisan, Kerem Ege and Bernard Laulagnet, JASA, p. 39

For the strain energy, we must calculate the small deformation tensor $\overline{\overline{\boldsymbol{\epsilon}}}=\frac{1}{2}\left(\operatorname{gra} \bar{a} d(\overline{\boldsymbol{U}})+\operatorname{gra} \bar{a} d(\overline{\boldsymbol{U}})^{T}\right)$ and the strain tensor $\overline{\boldsymbol{\epsilon}}=\overline{\bar{M}}_{S} \overline{\boldsymbol{\sigma}}$ (Inverse Hook law). We find the following small deformation tensor and its vector form:

$$
\overline{\overline{\boldsymbol{\epsilon}}}=\frac{1}{2}\left|\begin{array}{ccc}
0 & -z w_{, x y}\left(x_{r}, y, t\right) & 0 \\
& -2 z w_{, y y}\left(x_{r}, y, t\right) & x w_{, x y}\left(x_{r}, y, t\right) \\
(\text { sym }) & 0
\end{array}\right| \Longleftrightarrow \overline{\boldsymbol{\epsilon}}=\frac{1}{2}\left|\begin{array}{c}
0 \\
-2 z w_{, y y}\left(x_{r}, y, t\right) \\
0 \\
-z w_{, x y}\left(x_{r}, y, t\right) \\
0 \\
x w_{, x y}\left(x_{r}, y, t\right)
\end{array}\right|
$$

The strains are linked to deformations through a flexibility matrix. The matrix $\overline{\bar{M}}_{S}$ for an orthotropic material is:

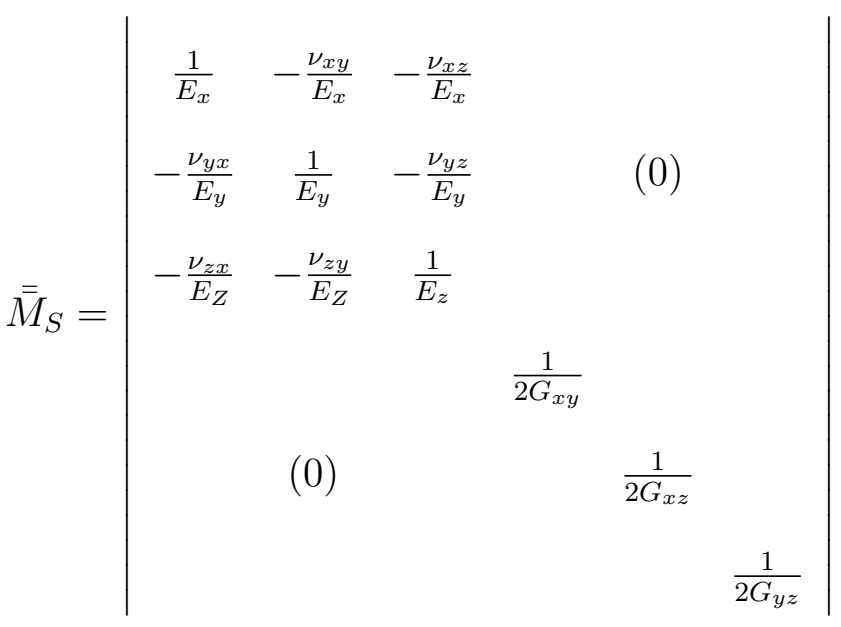


B. Trévisan, Kerem Ege and Bernard Laulagnet, JASA, p. 40

Thus we determine the three strains in the rib:

$$
\left\{\begin{array}{c}
\sigma_{y y}=-z E_{y} w_{, y y}\left(x_{r}, y, t\right) \\
\sigma_{x y}=-z G_{x y} w_{, x y}\left(x_{r}, y, t\right) \\
\sigma_{y z}=x G_{y z} w_{, x y}\left(x_{r}, y, t\right)
\end{array}\right.
$$

After which we calculate the strain energy:

$$
E_{D}=\frac{1}{2} \int_{x=-\frac{b}{2}}^{\frac{b}{2}} \int_{y=0}^{L} \int_{h=\frac{h}{2}}^{\frac{b}{2}+H} \boldsymbol{\sigma}: \boldsymbol{\epsilon} d x d y d z
$$

with $\sigma: \epsilon=E_{y} z^{2} w_{, y y}^{2}\left(x_{r}, y, t\right)+\left(G_{x y} z^{2}+G_{y z} x^{2}\right) w_{, x y}^{2}\left(x_{r}, y, t\right)$, let :

$$
E_{D}=\frac{1}{2} \int_{y=0}^{L} E_{y} I_{f} w_{, y y}^{2}\left(x_{r}, y, t\right)+D_{g} w_{, x y}^{2}\left(x_{r}, y, t\right) d y
$$

with $I_{f}=\frac{b}{3}\left[\left(\frac{h}{2}+H\right)^{3}-\left(\frac{h}{2}\right)^{3}\right]$ and $D_{g}=\int_{x=-\frac{b}{2}}^{\frac{b}{2}} \int_{h=\frac{h}{2}}^{\frac{b}{2}+H} G_{x y} z^{2}+G_{y z} x^{2} d x d z=G_{y z} \frac{H b^{3}}{12}+G_{x y} I_{f}$ that we define as the torsion dynamic rigidity of an orthotropic beam in the plane $(\vec{x}, \vec{z})$.

For the sake of simplicity, we consider the beam as isotropic in the following. $D_{g}$ becomes $D_{g}=G\left(\frac{H b^{3}}{12}+I_{f}\right)=G_{r} I_{g}$. So, we express the Hamilton function of the rib $H_{r}$ :

$$
\begin{gathered}
H_{r}=\int_{t_{0}}^{t_{1}} \frac{1}{2} \int_{y=0}^{L} \rho_{r}\left(I_{f} \dot{w}_{, y}^{2}\left(x_{r}, y, t\right)+b H \dot{w}^{2}\left(x_{r}, y, t\right)+I_{g} \dot{w}_{, x}^{2}\left(x_{r}, y, t\right)\right) \\
-E_{r} I_{f} w_{, y y}^{2}\left(x_{r}, y, t\right)+G_{r} I_{g} w_{, x y}^{2}\left(x_{r}, y, t\right) d y d t
\end{gathered}
$$


B. Trévisan, Kerem Ege and Bernard Laulagnet, JASA, p. 41

that we gather on a line at $x=x_{r}$ :

$$
\begin{gathered}
H_{r}=\int_{t_{0}}^{t_{1}} \frac{1}{2} \int_{x=0}^{l} \int_{y=0}^{L}\left[\rho_{r}\left(I_{f} \dot{w}_{, y}^{2}(x, y, t)+b H \dot{w}^{2}(x, y, t)\right)-E_{r} I_{f} w_{, y y}^{2}(x, y, t)\right] \delta\left(x-x_{r}\right) d x d y d t \\
+\int_{t_{0}}^{t_{1}} \frac{1}{2} \int_{x=0}^{l} \int_{y=0}^{L}\left[\rho_{r} I_{g} \dot{w}_{, x}^{2}(x, y, t)+G_{r} I_{g} w_{, x y}^{2}(x, y, t)\right] \delta\left(x-x_{r}\right) d x d y d t \\
=H_{r b}+H_{r t}
\end{gathered}
$$

For the straight rib, the functional $H_{r}$ is determined following the same approach so we obtain:

$$
\begin{gathered}
H_{b}=\int_{t_{0}}^{t_{1}} \frac{1}{2} \int_{x=0}^{l} \int_{y=0}^{L}\left[\rho_{c}\left(I_{c} \dot{w}_{, x}^{2}(x, y, t)+b_{c} H_{c} \dot{w}^{2}(x, y, t)\right)-E_{c} I_{f c} w_{, x x}^{2}(x, y, t)\right] \delta\left(y-y_{c}\right) d x d y d t \\
+\int_{t_{0}}^{t_{1}} \frac{1}{2} \int_{x=0}^{l} \int_{y=0}^{L}\left[\rho_{c} I_{g c} \dot{w}_{, y}^{2}(x, y, t)+G_{c} I_{g c} w_{, x y}^{2}(x, y, t)\right] \delta\left(y-y_{c}\right) d x d y d t \\
=H_{b b}+H_{b t}
\end{gathered}
$$


B. Trévisan, Kerem Ege and Bernard Laulagnet, JASA, p. 42

a)

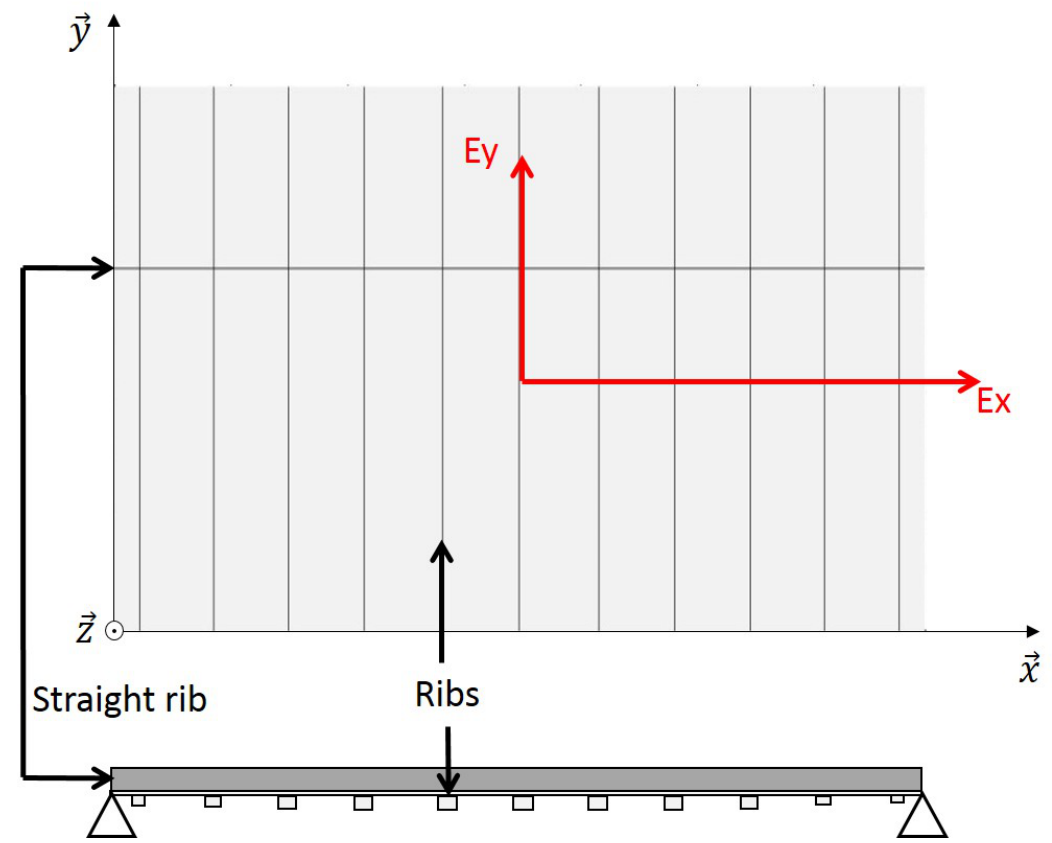

Figure 1: (a) top view of the stiffened rectangular wood panel ; (b) section view of the panel. 
B. Trévisan, Kerem Ege and Bernard Laulagnet, JASA, p. 43

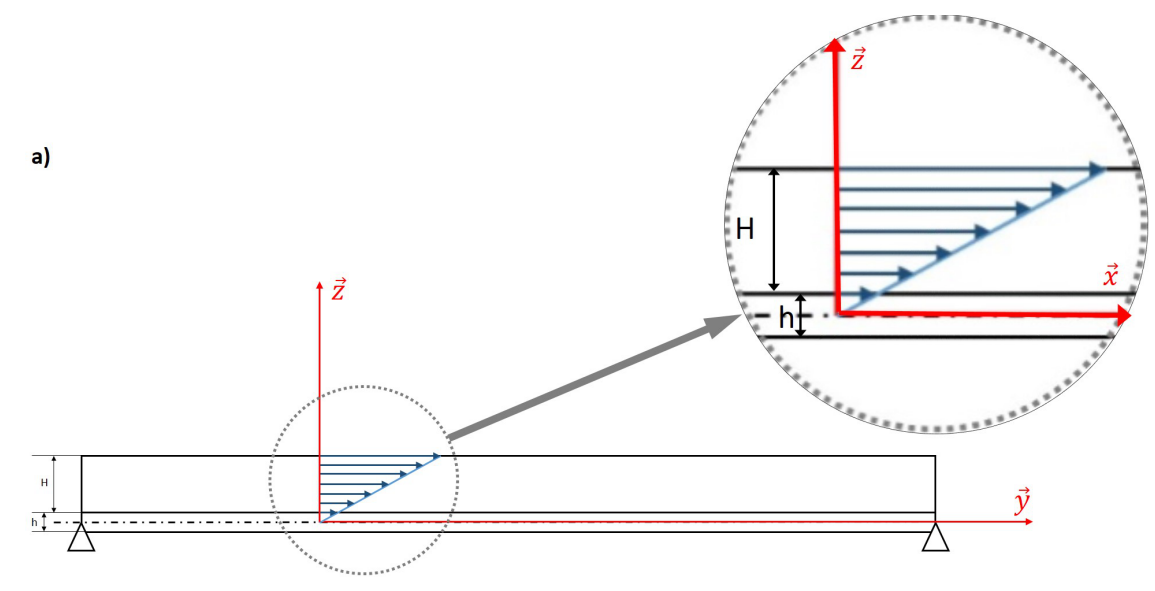

b)

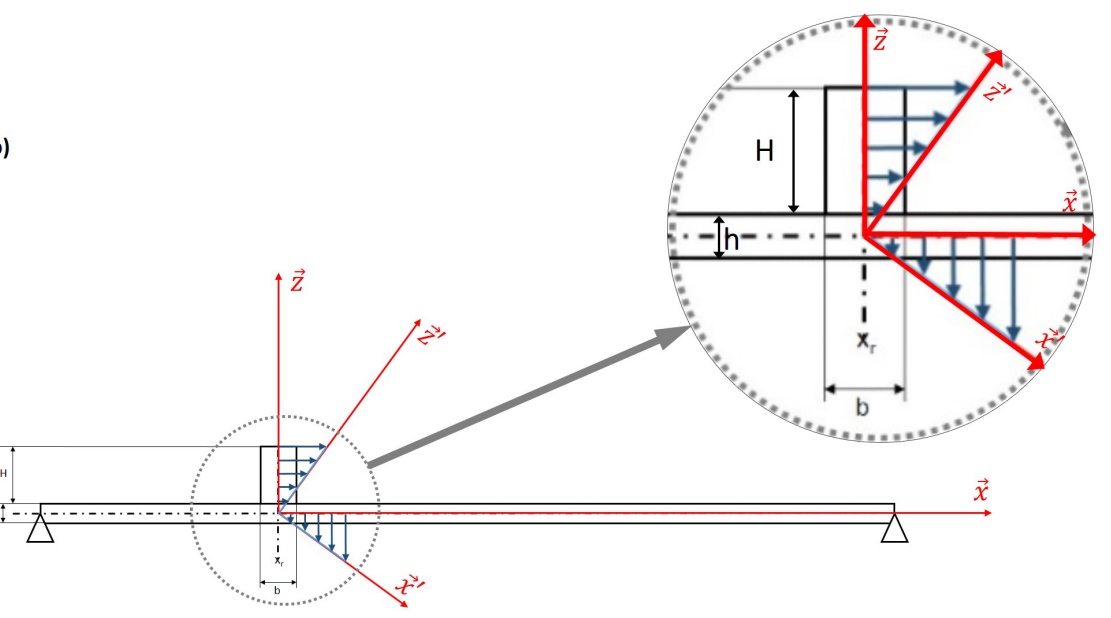

Figure 2: Diagrams of displacement continuity at the interface plate / superstructure along $\vec{y}$. (a) bending plane; (b) torsion plane. 
B. Trévisan, Kerem Ege and Bernard Laulagnet, JASA, p. 44

a)
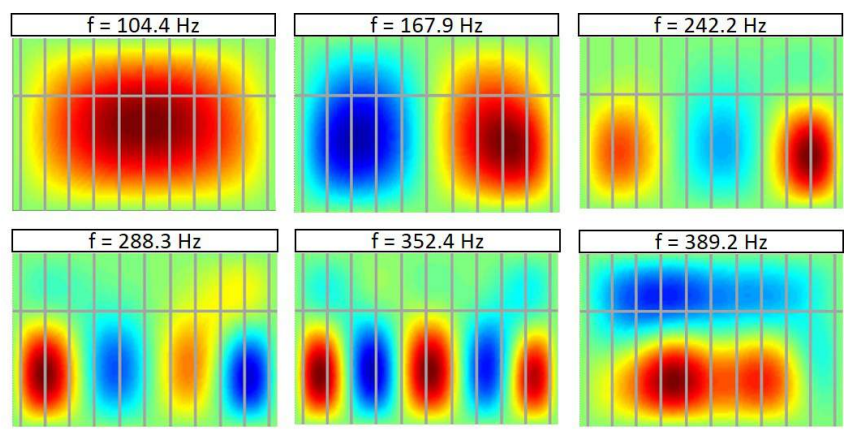

b)

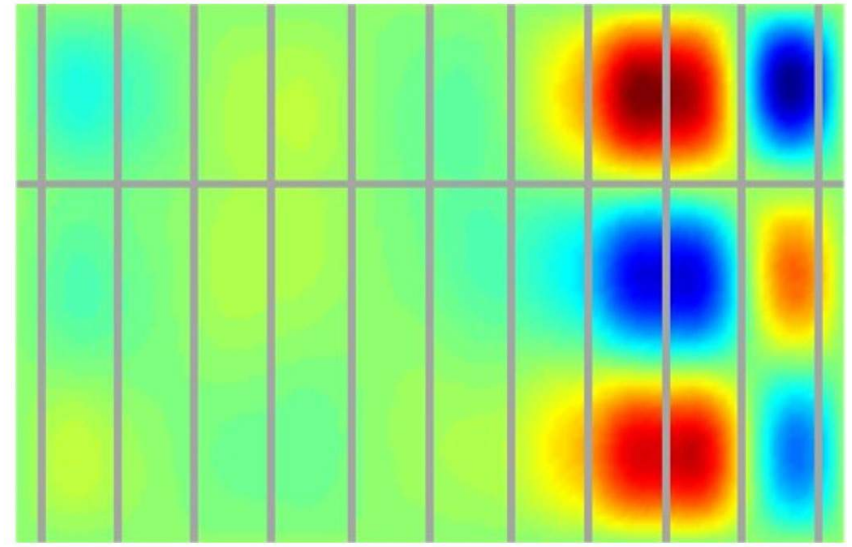

c)

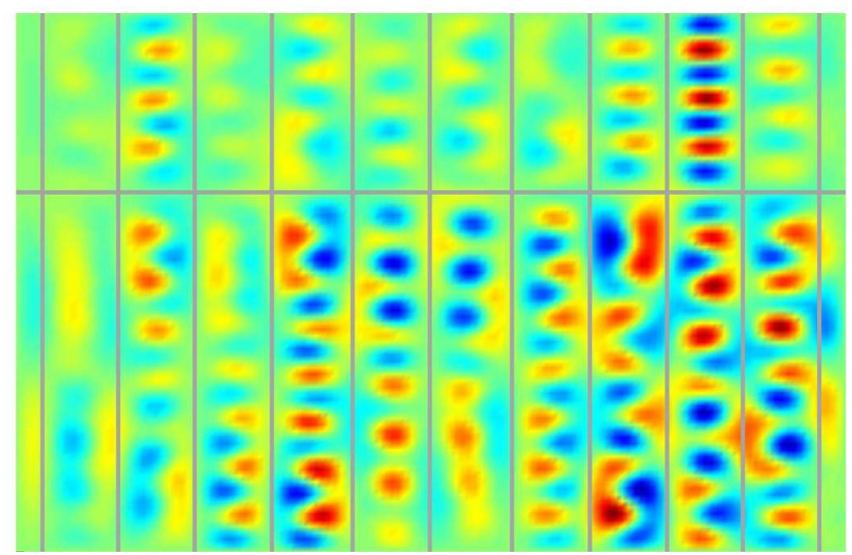

Figure 3: Modes of the stiffened rectangular wood panel. (a) $1^{\text {st }}$ to $6^{\text {th }}$ modes (low frequencies); (b): $17^{\text {th }}$ mode at $865.8 \mathrm{~Hz}$ with localized vibratory phenomena; (c) $271^{\text {th }}$ mode at $4190 \mathrm{~Hz}$ with complex vibratory phenomena. 
B. Trévisan, Kerem Ege and Bernard Laulagnet, JASA, p. 45
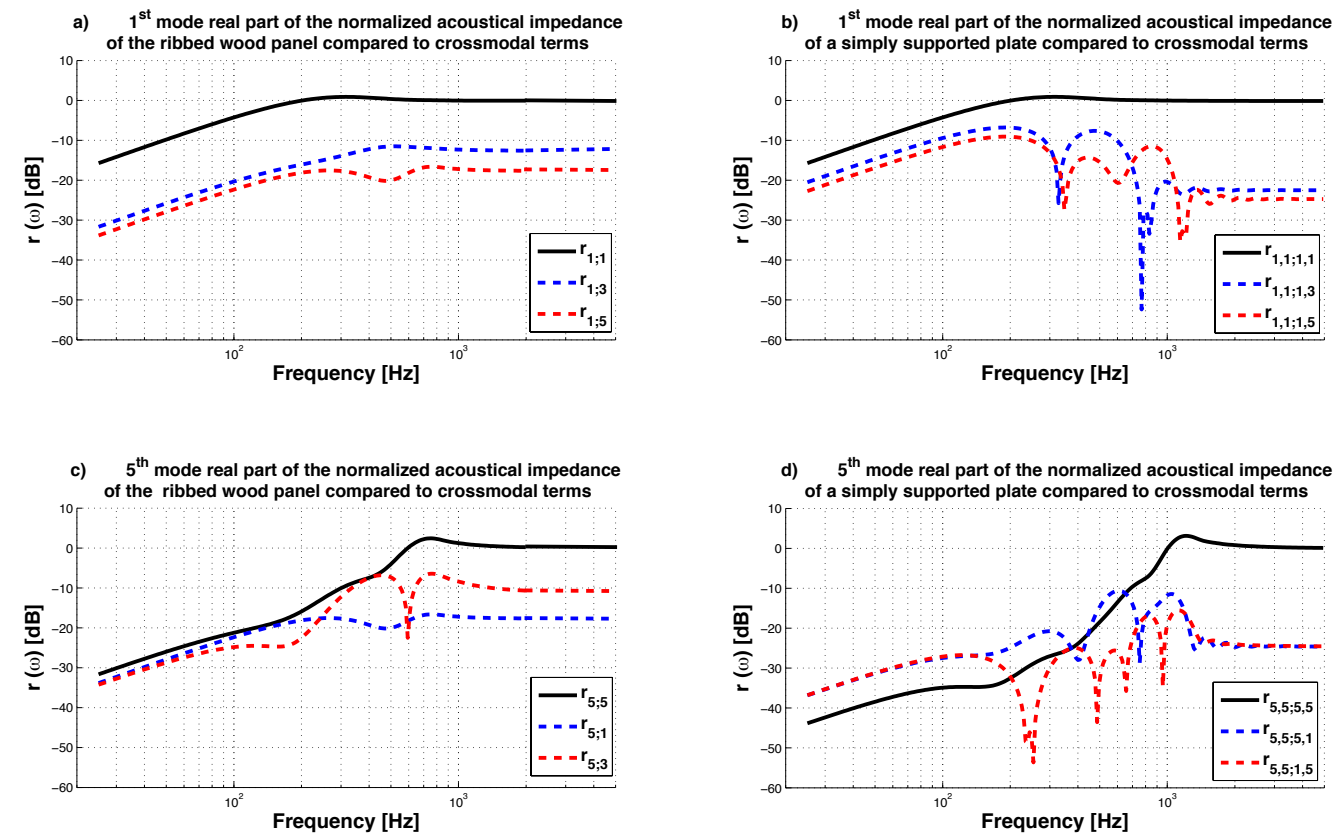

Figure 4: Real parts of normalized radiation impedances of the $1^{\text {st }}$ (top: fig a) and $5^{\text {th }}$ (bottom: fig c) and d) modes for two structures: on left (fig a) and c)) for the stiffened rectangular wood panel ; on right (fig b) and d) for a simply supported plate. 
B. Trévisan, Kerem Ege and Bernard Laulagnet, JASA, p. 46

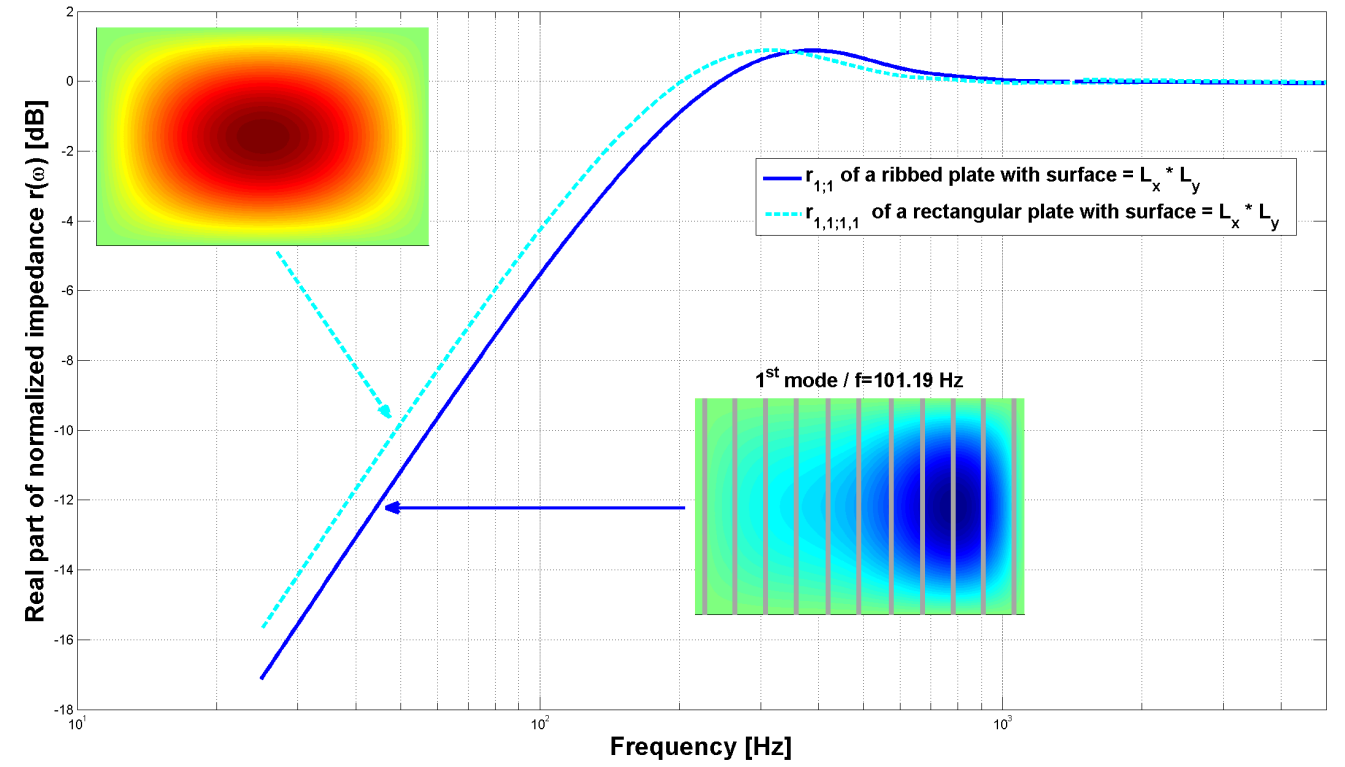

Figure 5: Normalized radiation coefficient of the $1^{\text {st }}$ mode of a rectangular wood panel ribbed in one direction. 
B. Trévisan, Kerem Ege and Bernard Laulagnet, JASA, p. 47

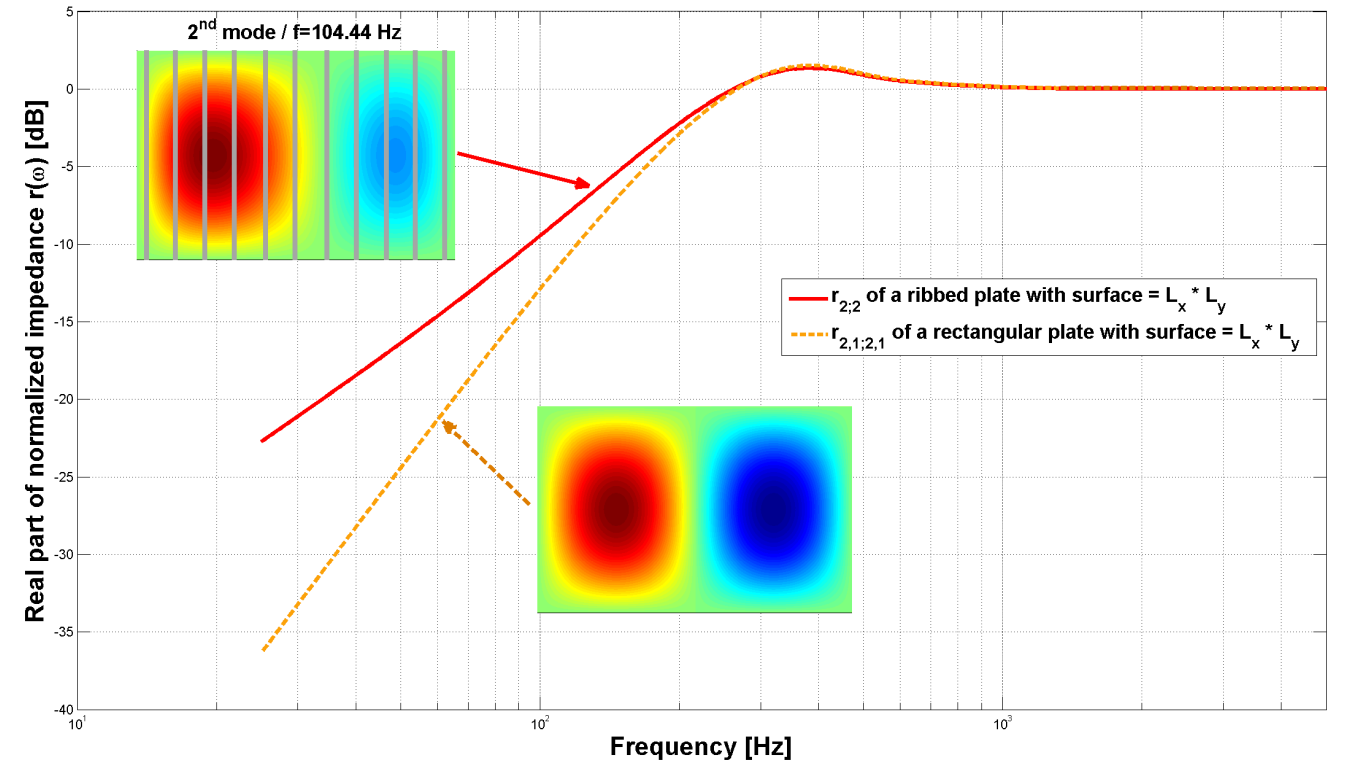

Figure 6: Normalized radiation coefficient of the $2^{\text {nd }}$ mode of a rectangular wood panel ribbed in one direction. 
B. Trévisan, Kerem Ege and Bernard Laulagnet, JASA, p. 48

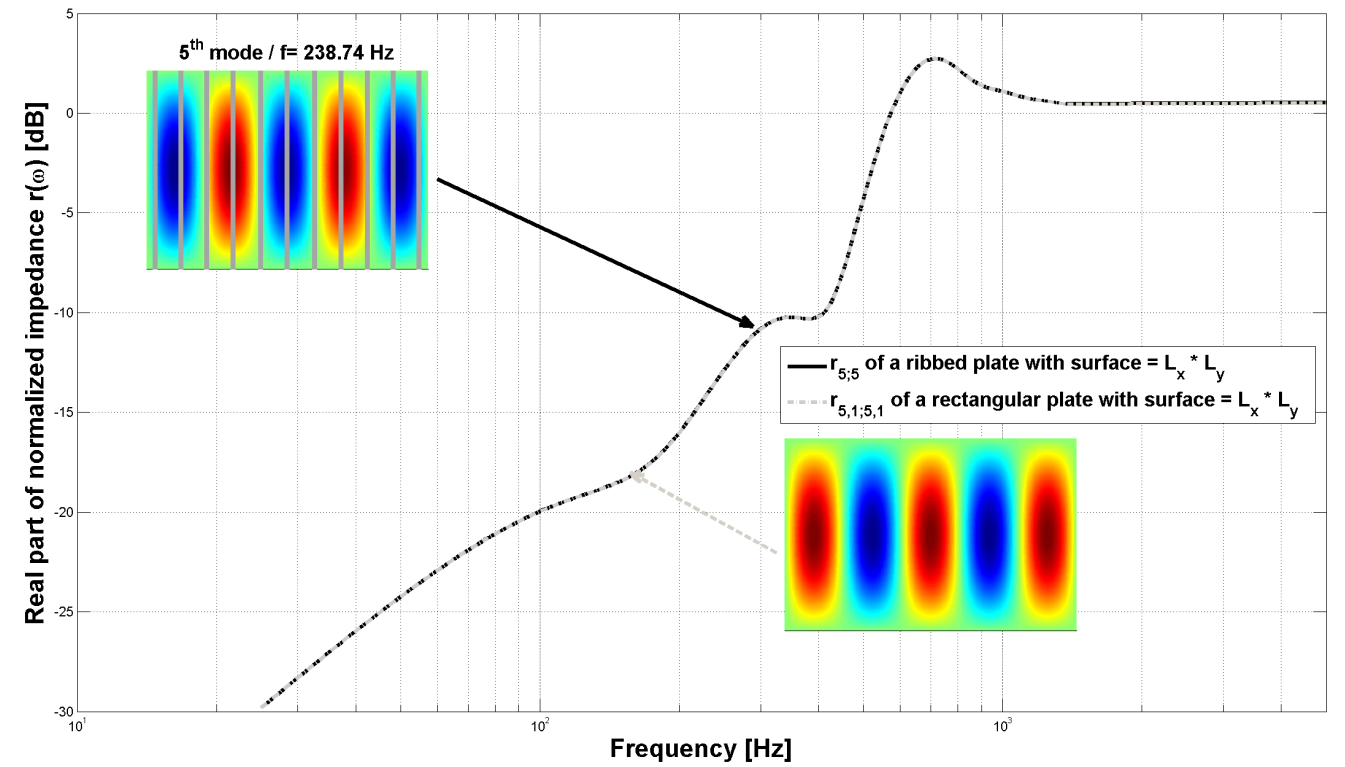

Figure 7: Normalized radiation coefficient of the $5^{\text {th }}$ mode of a rectangular wood panel ribbed in one direction. 
B. Trévisan, Kerem Ege and Bernard Laulagnet, JASA, p. 49

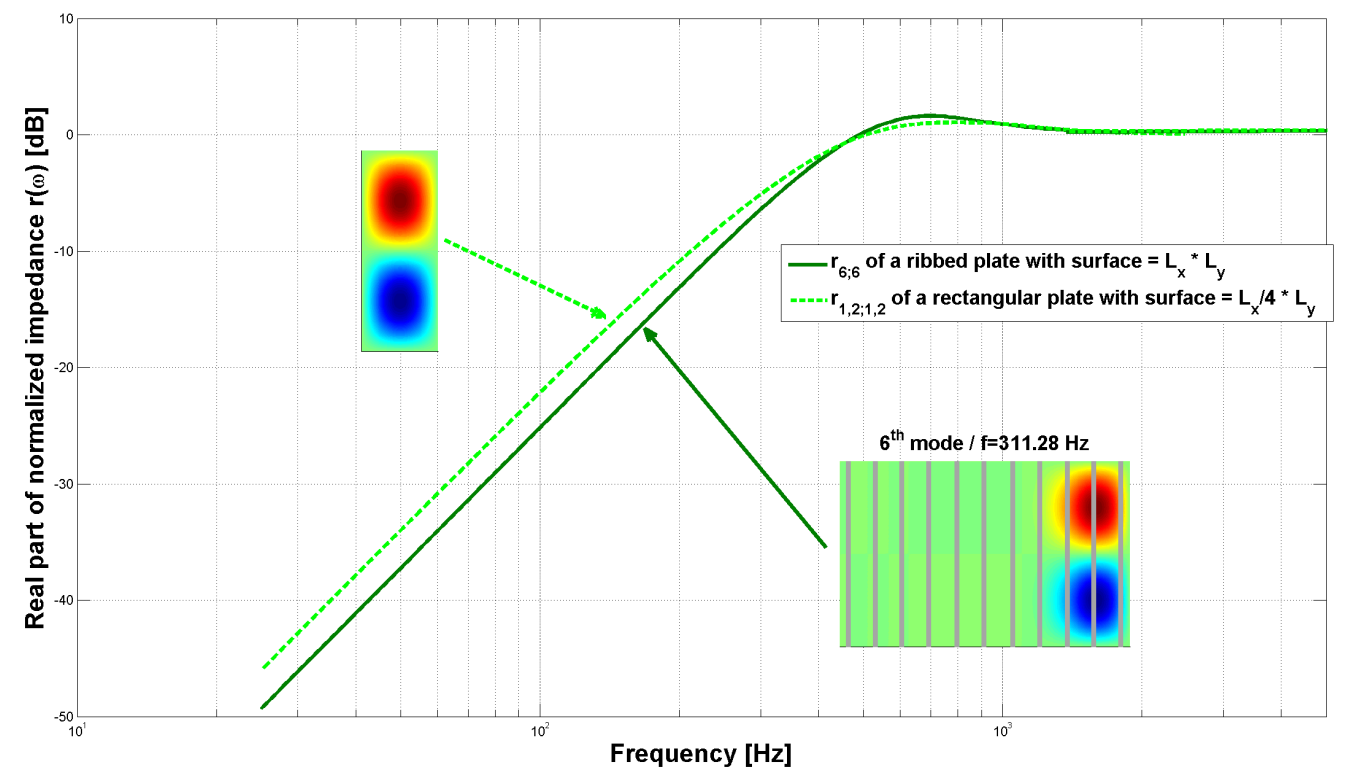

Figure 8: Normalized radiation coefficient of the $6^{\text {th }}$ mode of a rectangular wood panel ribbed in one direction. 
B. Trévisan, Kerem Ege and Bernard Laulagnet, JASA, p. 50

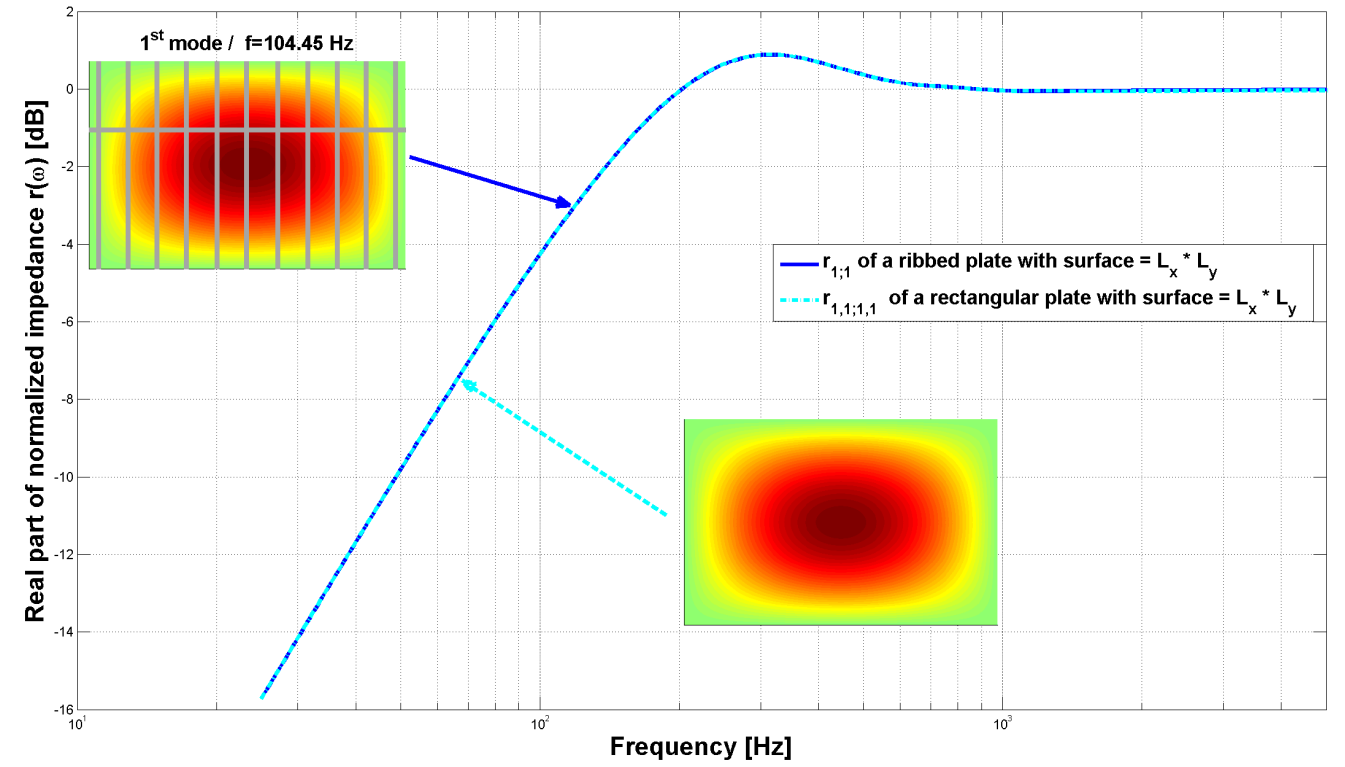

Figure 9: Normalized radiation coefficient of the $1^{\text {st }}$ mode of a rectangular wood panel ribbed in two directions. 
B. Trévisan, Kerem Ege and Bernard Laulagnet, JASA, p. 51

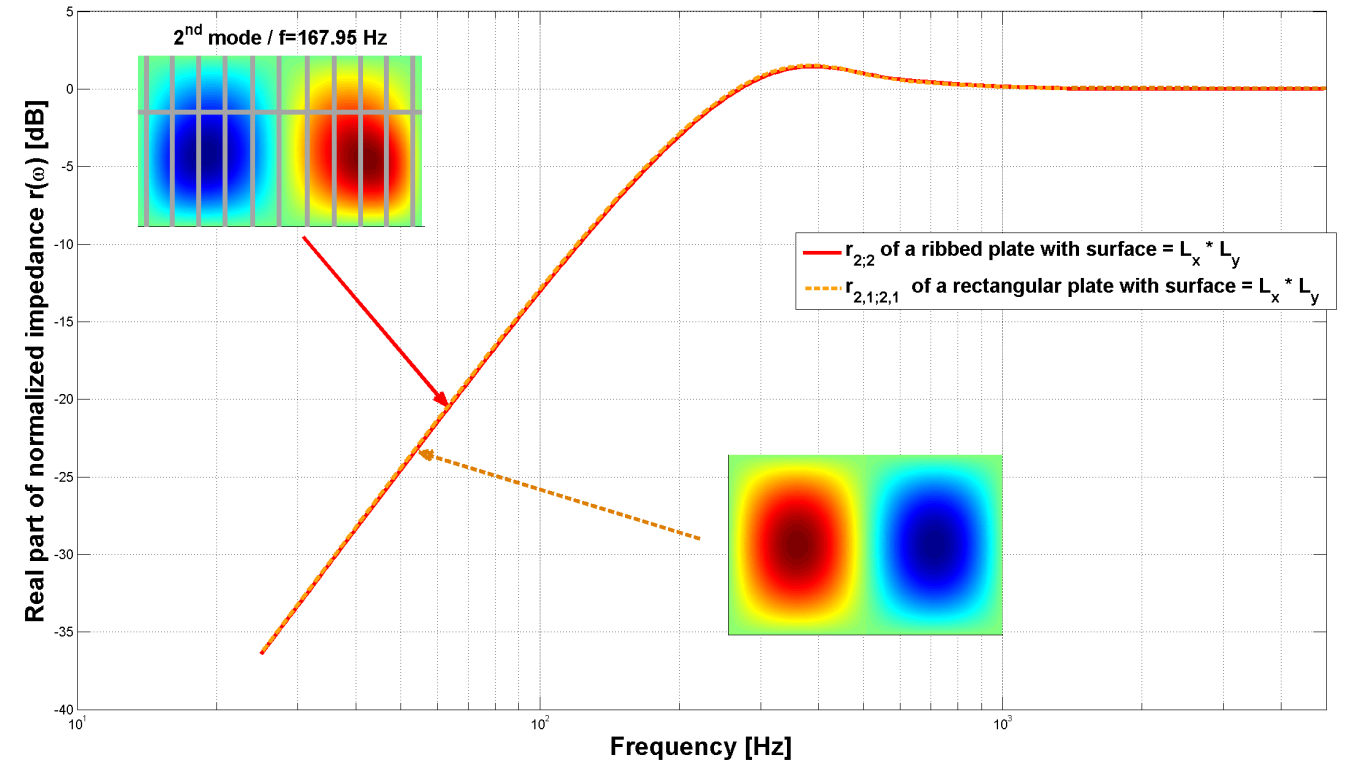

Figure 10: Normalized radiation coefficient of the $2^{\text {nd }}$ mode of a rectangular wood panel ribbed in two directions. 
B. Trévisan, Kerem Ege and Bernard Laulagnet, JASA, p. 52

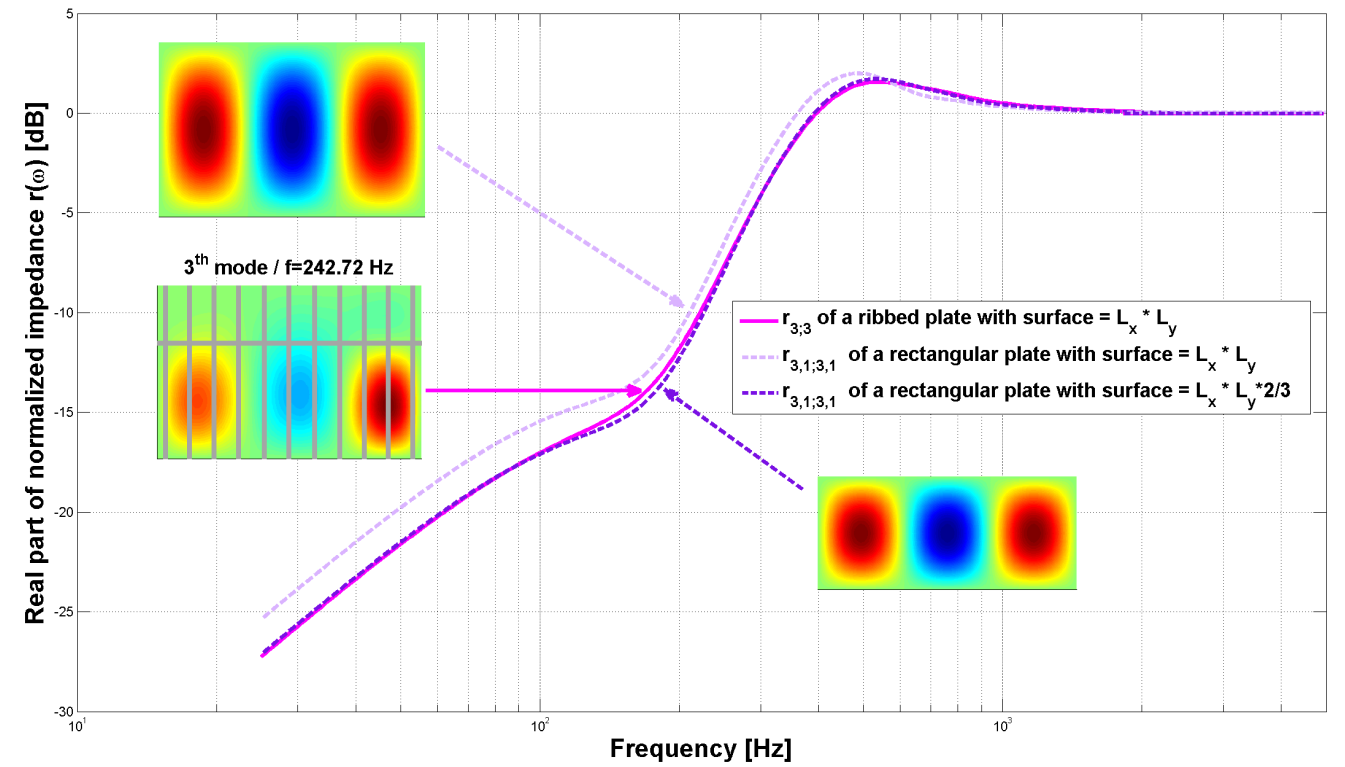

Figure 11: Normalized radiation coefficient of the $3^{\text {th }}$ mode of a rectangular wood panel ribbed in two directions. 
B. Trévisan, Kerem Ege and Bernard Laulagnet, JASA, p. 53

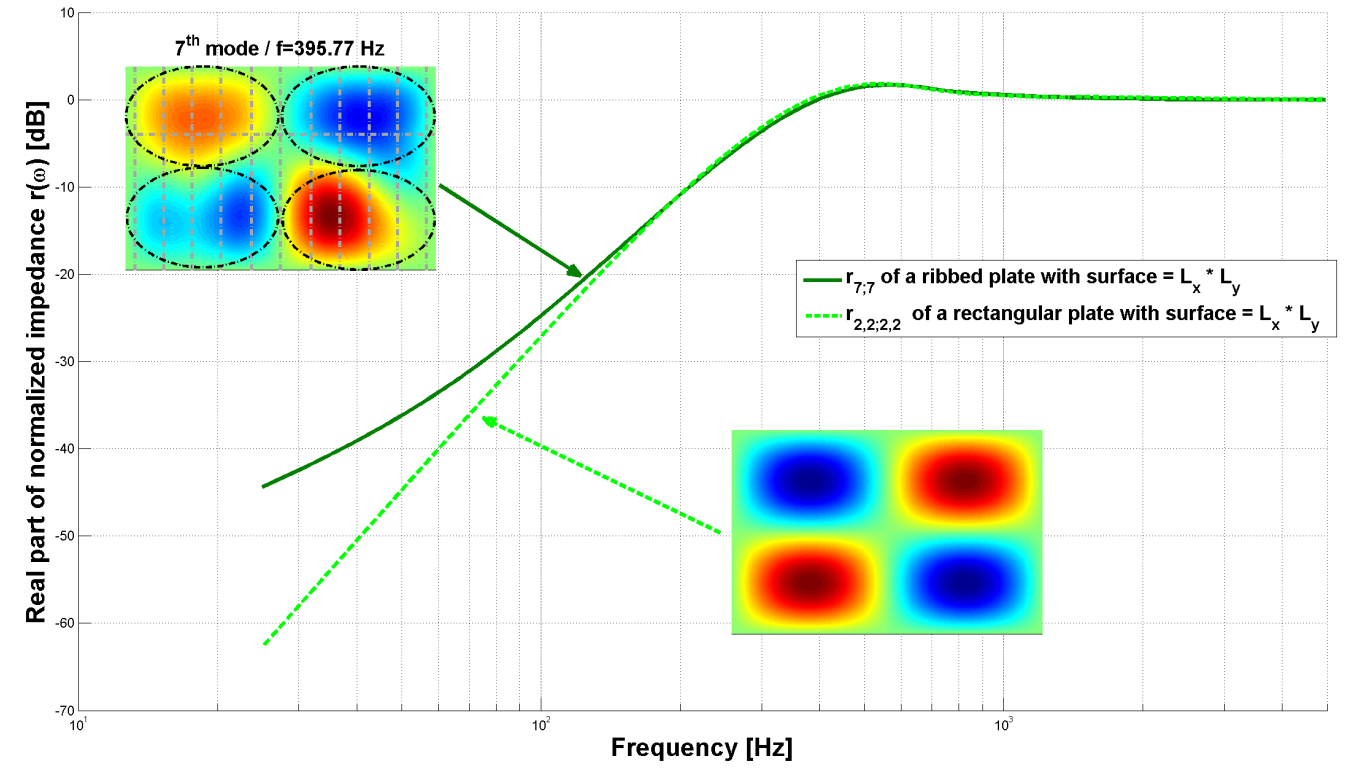

Figure 12: Normalized radiation coefficient of the $7^{\text {th }}$ mode of a rectangular wood panel ribbed in two directions. 
B. Trévisan, Kerem Ege and Bernard Laulagnet, JASA, p. 54

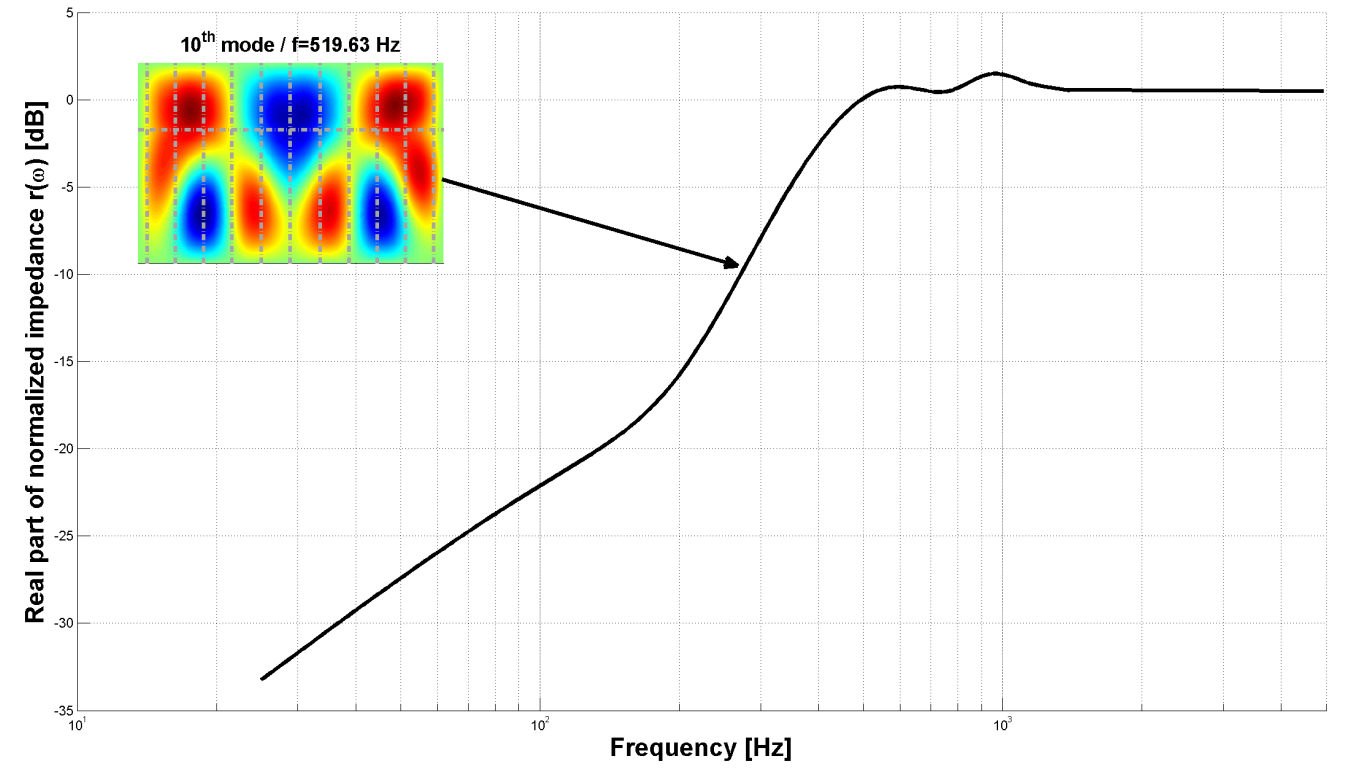

Figure 13: Normalized radiation coefficient of the $10^{\text {th }}$ mode a rectangular wood panel ribbed in two directions. 
B. Trévisan, Kerem Ege and Bernard Laulagnet, JASA, p. 55

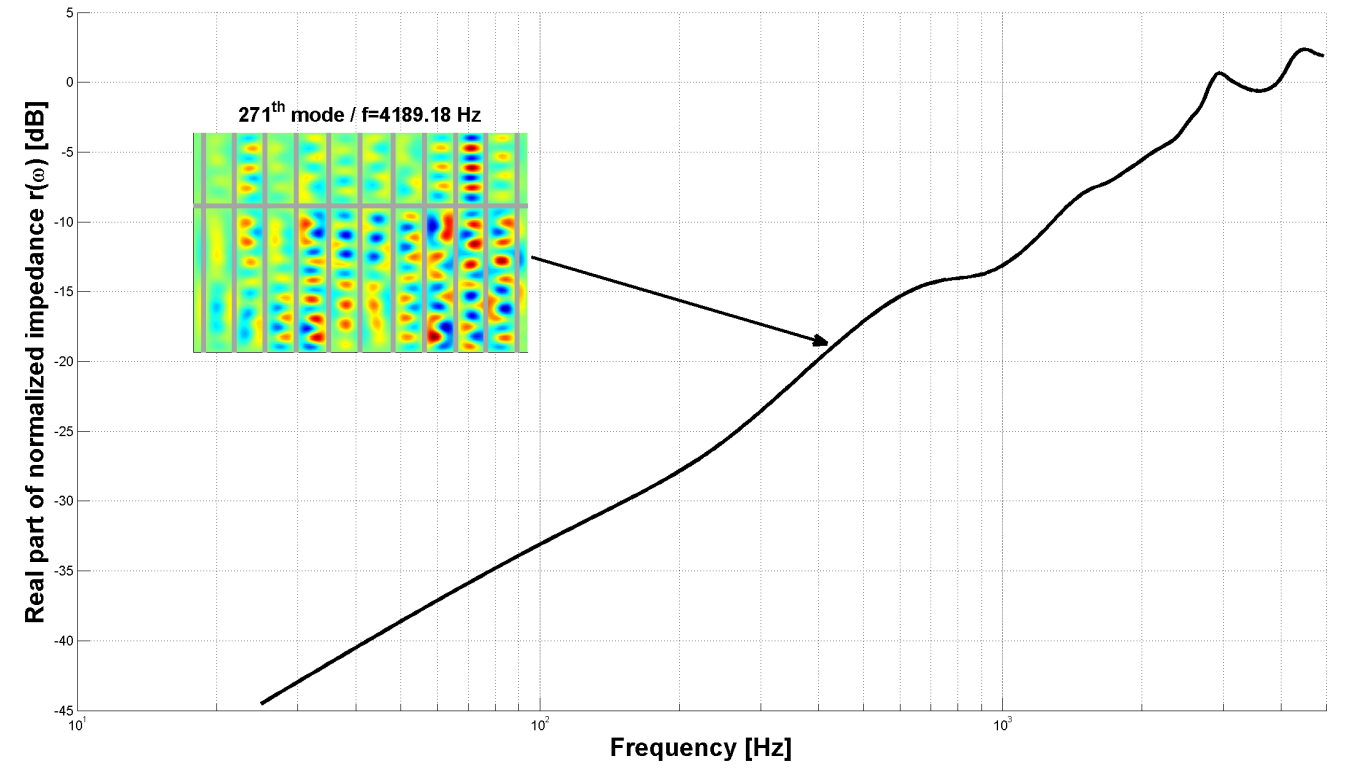

Figure 14: Normalized radiation coefficient of the $271^{\text {th }}$ mode of a rectangular wood panel ribbed in two directions. 
B. Trévisan, Kerem Ege and Bernard Laulagnet, JASA, p. 56
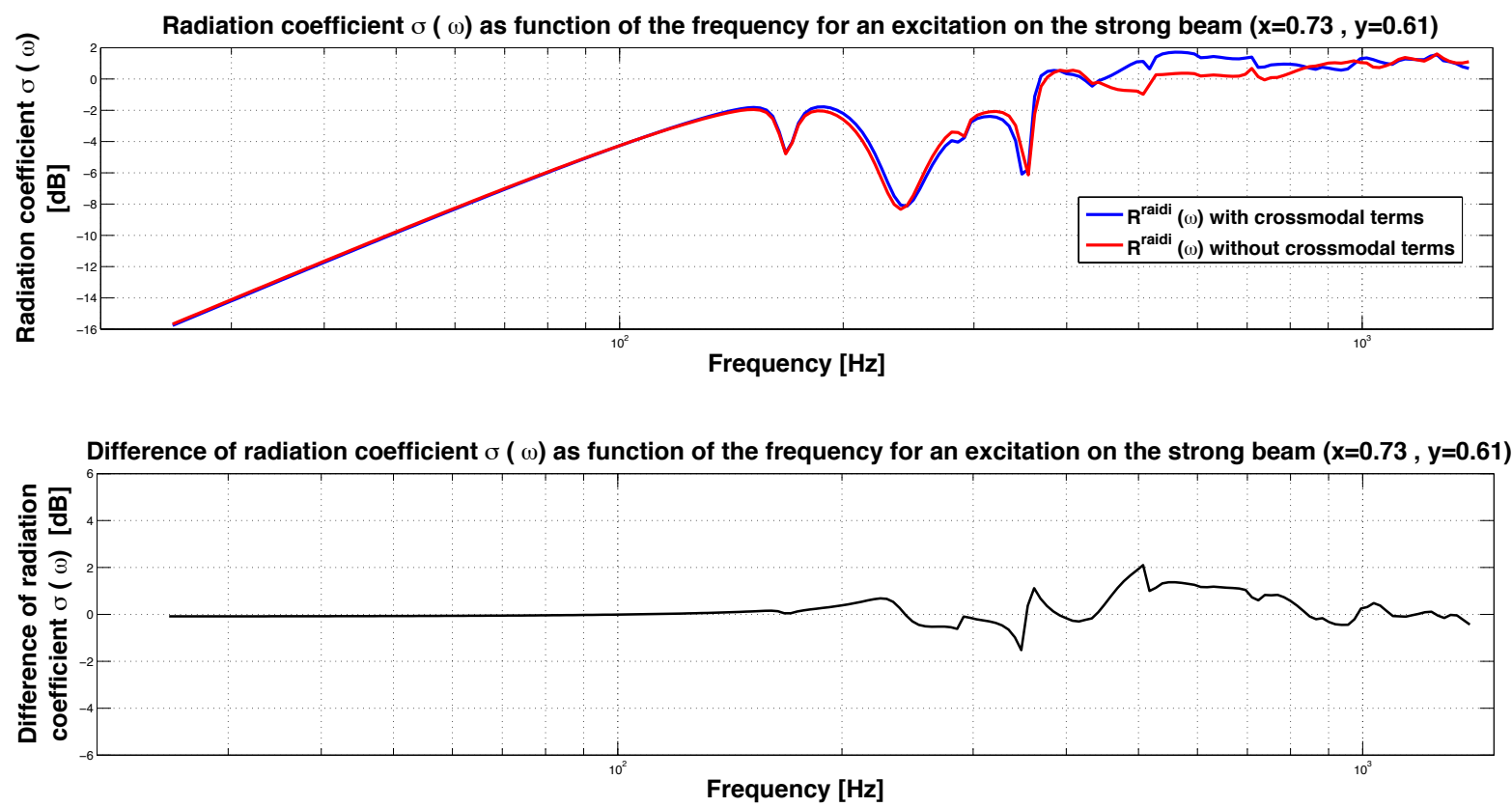

Figure 15: Normalized radiation coefficient with and without taking into account crossmodal terms in $R^{\text {ribbed }}(\omega)$ : top: radiation coefficient $\sigma(\omega)$; bottom: difference between the two plots. 
B. Trévisan, Kerem Ege and Bernard Laulagnet, JASA, p. 57

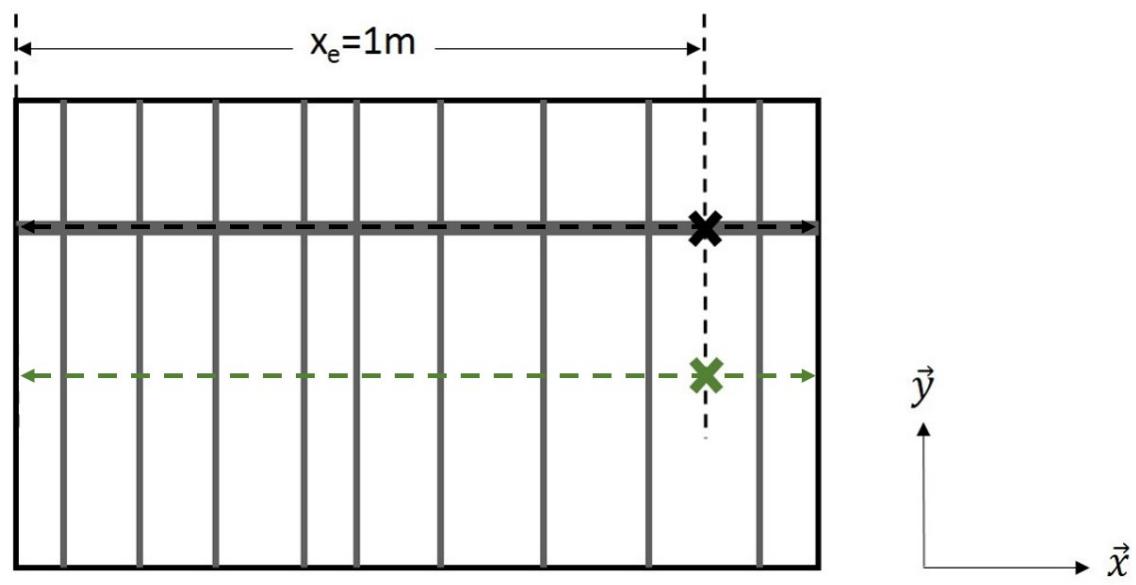

Figure 16: Drawing of the positions of the two excitation points. 
B. Trévisan, Kerem Ege and Bernard Laulagnet, JASA, p. 58

a)

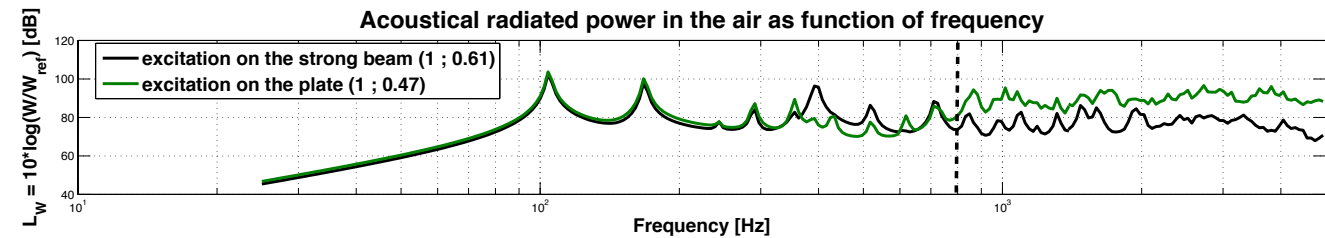

b)
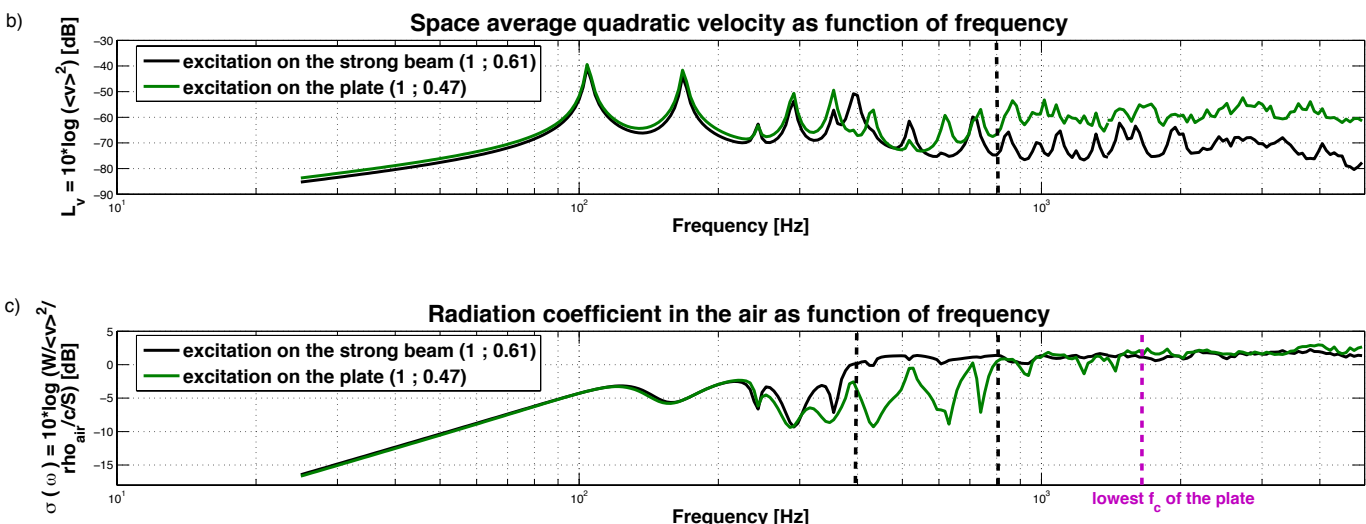

Figure 17: Acoustical radiated power, space average quadratic velocity and radiation coef-

ficient as a function of frequency for two excitations points on a stiffened rectangular wood panel: in full plot on the straight rib, in dashed plot far from the straight rib. 
B. Trévisan, Kerem Ege and Bernard Laulagnet, JASA, p. 59

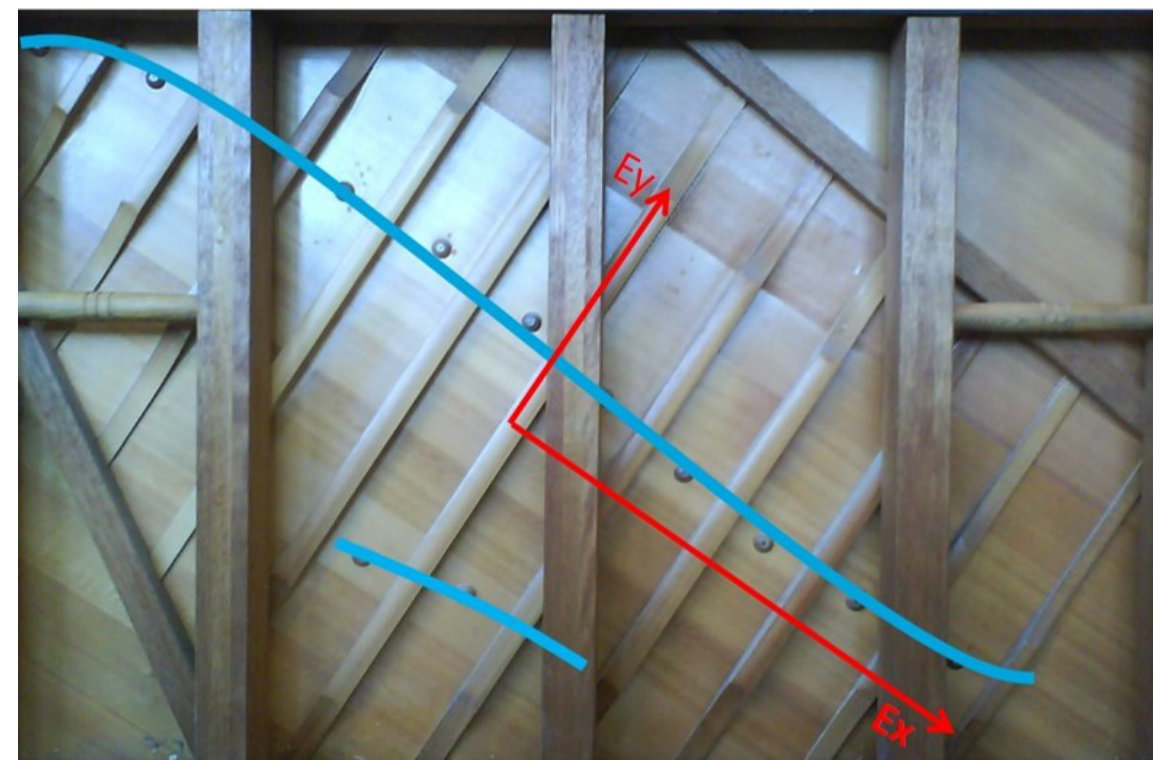

Figure 18: Upright piano soundboard from ${ }^{39}$. 
B. Trévisan, Kerem Ege and Bernard Laulagnet, JASA, p. 60

a)

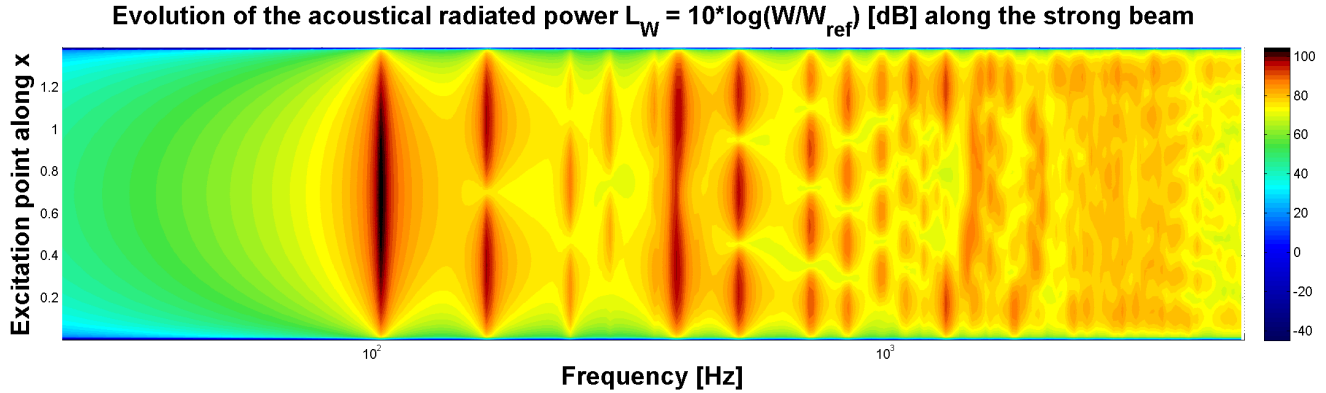

Evolution of the acoustical radiated power $L_{w}=10^{\star} \log \left(W / W_{\text {ref }}\right)[\mathrm{dB}]$ far from the strong beam along $x$

b)

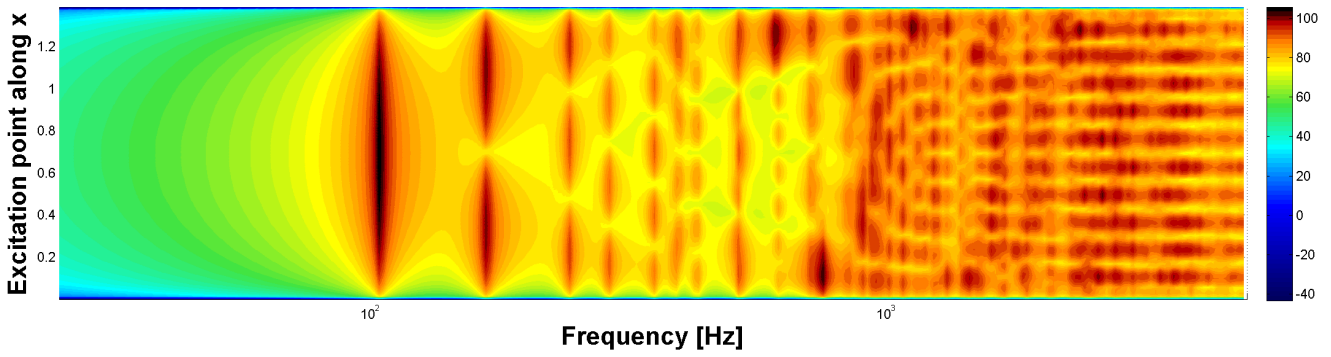

Figure 19: Evolution of the acoustical radiated power $L_{W}=10 \log \left(W / W_{\text {ref }}\right)[\mathrm{dB}]$ as a function of frequency for a stiffened rectangular wood panel. Top: along the straight rib ; bottom: far from the straight rib. 
B. Trévisan, Kerem Ege and Bernard Laulagnet, JASA, p. 61

\begin{tabular}{|c|l||c|l|}
\hline$l$ & Plate length & $L$ & Plate width \\
\hline$\rho$ & Plate mass density & $h$ & Plate thickness \\
\hline$D_{i}$ & Plate dynamic stiffness & $\rho_{r}$ & Rib mass density \\
\hline$E_{r}$ & Rib Young's modulus & $H$ & Rib height \\
\hline$b$ & Rib width & $I_{f}$ & Rib momentum of bending inertia \\
\hline$G_{r}$ & Rib shear modulus & $I_{g}$ & Rib momentum of torsion inertia \\
\hline$E_{c}$ & Straight rib Young's modulus & $H_{c}$ & Straight rib height \\
\hline$b_{c}$ & Straight rib width & $I_{f c}$ & Straight rib momentum of bending inertia \\
\hline$G_{c}$ & Straight rib shear modulus & $I_{g c}$ & Straight rib momentum of torsion inertia \\
\hline$\rho_{c}$ & Straight rib mass density & $H_{p}$ & Plate Hamilton functional \\
\hline$H_{r b}$ & Rib bending Hamilton functional & $H_{r t}$ & Rib torsion Hamilton functional \\
\hline$H_{b b}$ & Straight rib bending Hamilton functional & $H_{b t}$ & Straight rib torsion Hamilton functional \\
\hline
\end{tabular}

Table 1: Description of the different constants used in the Hamilton function of the problem. 
B. Trévisan, Kerem Ege and Bernard Laulagnet, JASA, p. 62

\begin{tabular}{|c|c|}
\hline$W(\omega)$ & Acoustical radiated power \\
\hline$<v^{2}>(\omega)$ & Space average quadratic velocity \\
\hline$\sigma(\omega)$ & Radiation coefficient \\
\hline$\left(x_{e}, y_{e}\right)$ & Coordinates of the excitation point \\
\hline $\bar{F}_{g e n}$ & Generalized force vector \\
\hline$F_{p q}$ & Component of $\bar{F}_{g e n}$ \\
\hline $\bar{a}$ & Vector of modal amplitudes in the simply supported plate basis \\
\hline $\bar{b}$ & Vector of modal amplitudes in the ribbed basis \\
\hline$\eta$ & Structural damping \\
\hline$p(x, y, z=0)$ & Acoustic boundary pressure \\
\hline$u_{z}$ & Particular acoustical displacement $\vec{z}$ \\
\hline$S$ & Plate surface \\
\hline$c$ & Acoustical celerity \\
\hline$\rho_{0}$ & Fluid mass density \\
\hline$\overline{\bar{Z}}^{a}(\omega)$ & Inter-modal acoustical impedance matrix \\
\hline$Z_{m n p q}(\omega)$ & Term of $\overline{\bar{Z}}^{a}(\omega)$ \\
\hline$\overline{\bar{R}}^{a}(\omega)$ & Real part of $\overline{\bar{Z}}^{a}$ \\
\hline$R_{m n p q}(\omega)$ & Real part of $Z_{m n p q}(\omega)$ \\
\hline$\overline{\bar{R}}^{s}(\omega)$ & Real part of the acoustical impedance matrix in the stiffened basis \\
\hline$r(\omega)$ & Normalized acoustical impedance \\
\hline$P_{p q}^{a}$ & Generalized acoustic pressure \\
\hline $\bar{P}_{a}$ & Vector of $P_{p q}^{a}$ \\
\hline$\overline{\bar{P}}$ & Transfer matrix \\
\hline$\tilde{\Gamma}\left(k_{x}, k_{y}\right)$ & Bi-dimensional Fourier transform of function $\Gamma(x, y)$ \\
\hline
\end{tabular}

Table 2: Acoustic radiation glossary 
B. Trévisan, Kerem Ege and Bernard Laulagnet, JASA, p. 63

\section{REFERENCES}

1. J. L. Guyader, Vibration in Continuous Media, London: ISTE Ltd., 2006.

2. A. W. Leissa, Vibration of plates, Scientific and Technical Information Division, National Aeronautics ans Space Administration, vol. 160 (1969).

3. B. Laulagnet, Sound radiation by a simply supported unbaffled plate, JASA, 103(5): 2451-2462 (1998).

4. D.J. Mead, A. K. Mallik, An approximate method of predicting the response of periodically supported beams subjected to random convected loading, JSV, 47(4), 457-471 (1976).

5. R. D. Mindlin, Influence of rotary inertia and shear on flexural motion of isotropic, elastic plate, Journal of Applied Mechanics-Transactions of the Asme, 18(1): 31-38 (1951).

6. C. Lesueur, Acoustic Radiation of Structures, vibroacoustic, fluid/structure interactions, Ed. Eyrolles, Paris (France), Parts 1, 2 \& 3 (1988).

7. P. R. Stepanischen, Modal coupling in the vibration of fluid loaded cylindrical shells, JASA, 71(4), 818-823 (1982). 
B. Trévisan, Kerem Ege and Bernard Laulagnet, JASA, p. 64

8. C. E. Wallace, Radiation Resistance of a Rectangular Panel, JASA, 51(3), 946-952 (1972).

9. A. Putra \& D. J. Thompson, Sound radiation from rectangular baffled and unbaffled plates, Applied Acoustics, 71(12), 1113-1125 (2010).

10. C. Wang, Modal sound transmission loss of a single leaf panel: Effects of inter-modal coupling, JASA, 137(6), 3514-3522 (2015).

11. G. Maidanik, Response of Ribbed Panels to Reverberant Acoustic Fields, JASA, 34(6), 809-826 (1962).

12. G. F. Lin, J. M. Garrelick, Sound transmission through periodically framed parallel plates, JASA, 61(4), 1014-1018 (1977).

13. D.J. Mead, S. Parthan, Free wave propagation in two dimensional periodic plates, $J S V$, 64(3), 325-348 (1979).

14. R. Vaicaitis, M. Slazak, Noise transmission through stiffened panels, JSV, 70(3), 413-426 (1980).

15. B. R. Mace, Periodically stiffened fluid-loaded plates, part 1: Response to convected harmonic pressure and free wave propagation, JSV, 73(4), 473-486 (1980). 
B. Trévisan, Kerem Ege and Bernard Laulagnet, JASA, p. 65

16. B. R. Mace, Periodically stiffened fluid-loaded plates, part 2 : Response to line and point forces, JSV, 73(4), 487-504 (1980).

17. B. R. Mace, Sound radiation from a plate reinforced by two sets of parallel stiffeners, JSV, 71(3), 435-441 (1980).

18. A. Mejdi \& N. Atalla, Dynamic and acoustic response of bidirectionally stiffened plates with eccentric stiffeners subject to airborne and structure-borne excitations, JSV, 329(21), 4422-4439 (2010).

19. J. Legault, A. Mejdi \& N. Atalla, Vibro-acoustic response of orthogonally stiffened panels: The effects of finite dimensions, JSV, 330(24), 5928-5948 (2011).

20. J. Legault \& N. Atalla, Sound transmission through a double panel structure periodically coupled with vibration insulator, JSV, 329(15), 3082-3100 (2010).

21. F.X. Xin, An exact elasticity model for rib-stiffened plates by decoupling acoustic coating layers, Composite Structures, 119, 559-567 (2015).

22. M. T. Chang, R. Vaicaitis, Noise transmission into semi cylindrical enclosures through discretely stiffened curved panels, JSV, 85(1), 71-83 (1982).

23. B. Laulagnet \& J. L. Guyader, Sound radiation by finite cylindrical ring stiffened shells, JSV, 138(2): 173-191 (1990). 
B. Trévisan, Kerem Ege and Bernard Laulagnet, JASA, p. 66

24. D. E. Beskos, J. B. Oates, Dynamic analysis of ring-stiffened circular cylindrical shells, JSV, 75(1), 1-15 (1981).

25. L. R. Koval, On sound transmission into a stiffened cylindrical shell with rings and stringers treated as discrete elements, JSV, 71(4), 511-521 (1980).

26. D.J. Mead, N. S. Bardell, Free vibration of a thin cylindrical shell with discrete axial stiffeners, JSV, 111(2), 229-250 (1986).

27. D.J. Mead, N. S. Bardell, Free vibration of a thin cylindrical shell with periodic circumferential stiffeners, JSV, 115(3), 499-520 (1987).

28. C. H. Hodges, J. Power, J. Woodhouse, The low frequency vibrations of a ribbed cylinder. Part one: Theory, JSV,101(2), 219-235 (1985).

29. C. H. Hodges, J. Power, J. Woodhouse, The low frequency vibrations of a ribbed cylinder. Part two: Observations and interpretations, JSV, 101(2), 237-256 (1985).

30. C. B. Burroughs, Acoustic radiation from fluid-loaded infinite circular cylinders with doubly periodic ring supports, JASA, 75(3), 715-722 (1984).

31. J. Chabassier,A. Chaigne and P. Joly, Modeling and simulation of a grand piano, JASA, 134(1), 648-665 (2013). 
B. Trévisan, Kerem Ege and Bernard Laulagnet, JASA, p. 67

32. A. Mejdi \& N. Atalla, Vibroacoustic analysis of laminated composite panels stiffened by complex laminated composite stiffeners, International Journal of Mechanical Sciences, 58(1), 13-26 (2012).

33. A. Mejdi, J. Legault \& N. Atalla, Transmission loss of periodically stiffened laminate composite panels: Shear deformation and in-plane interaction effects, JASA, 131(1), 174-185 (2012).

34. F.X. Xin \& T. J. Lu, Analytical modeling of fluid loaded orthogonally rib-stiffened sandwich structures: Sound Transmission, Journal of the Mechanics and Physics of Solids, 58(9), 1374-1396 (2010).

35. F.X. Xin \& T. J. Lu, Analytical modeling of wave propagation in orthogonally rib-stiffened sandwich structures: Sound radiation, Computers and Structures, 89(5-6), 507-516 (2011).

36. F.X. Xin \& T. J. Lu, Sound radiation of orthogonally rib-stiffened sandwich structures with cavity absorption, Composites Science and Technology, 70(15), 2198-2206 (2010).

37. F.X. Xin \& T. J. Lu, Transmission loss of orthogonally rib-stiffened double-panel structures with cavity absorption, JASA, 129(4), 1919-1934 (2011). 
B. Trévisan, Kerem Ege and Bernard Laulagnet, JASA, p. 68

38. J. Berthaut, M. N. Ichchou, \& L. Jezequel, Piano soundboard : structural behavior, numerical and experimental study in the modal range, Applied Acoustics, 64(1): 1113-1136 (2003).

39. K. Ege, X. Boutillon, \& M. Rébillat, Vibroacoustics of the piano soundboard : (Non)linearity and modal properties in the low- and mid-frequency ranges, JSV, 332(5): 1288-1305 (2013).

40. X. Boutillon \& K. Ege, Vibroacoustics of the piano soundboard : Reduced models, mobility synthesis, and acoustical radiation regime, JSV, 332(18): 4261-4279 (2013).

41. B. Laulagnet \& J. L. Guyader, Structural acoustic radiation prediction : expanding the vibratory response on a functional basis, Applied acoustics, 43(3): 247-269 (1994).

42. T. Gmür, Dynamics of structures. Numeric modal analysis, Presses polytechniques et universitaires romandes, 133-137 (1997).

43. E. G. Williams, Fourier Acoustics: Sound Radiation and Nearfield Acoustical Holography, Academic Press, Chapters 1 \& 2 (1999).

44. B.Laulagnet and J. L. Guyader in C.Valor, Validation commission of vibroacoustic calculation software packages: results of reference for the acoustic radiation of the 
B. Trévisan, Kerem Ege and Bernard Laulagnet, JASA, p. 69

plates, benchmarks for vibroacoustic software, Société française des mécaniciens /

French Society of Engineer, Courbevoie (France), 1-118 (1996).

45. B. Trévisan, K. Ege and B. Laulagnet, Prediction of orthotropic ribbed plates vibro-acoustics mechanisms: application to the piano soundboard, International Symposium on Musical Acoustics, Mans (France), 93 (2014).

46. B. Trévisan, K. Ege and B. Laulagnet, Piano soundboard vibro-acoustic modeling through decomposition on the basis of orthotropic simply supported unribbed plate, NOVEM : Noise and Vibration - Emerging Technologies, Dubrovnik (Croatia), 77 (2015).

47. H. Suzuki, Acoustics of Pianos, Applied Acoustics, 30(2): 147-205 (1990).

48. H. A. Conklin, Design and tone in the mechanoacoustic piano. Part II. Piano structure, JASA, 100(2): 695-708 (1996).

49. N. Giordano, Sound production by a vibrating piano soundboard: Experiment, JASA, 104(3):1648-1653 (1998).

50. N. Giordano, Mechanical impedance of a piano soundboard, JASA, 104(4): 2128-2133 (1998).

51. N. Giordano, Simple model of a piano soundboard, JASA, 102(2): 11591168 (1997). 
B. Trévisan, Kerem Ege and Bernard Laulagnet, JASA, p. 70

52. A. Chaigne, B. Cotté \& R. Viggiano, Dynamical properties of piano soundboards, JASA, 133(4): 2456-2466 (2013).

53. X. Boutillon, Model for piano hammers: Experimental determination and digital simulation, JASA, 83(2): 746-754 (1988). 